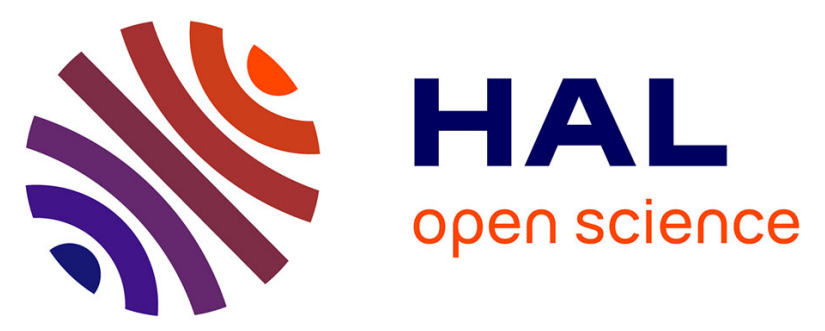

\title{
High Frame Rate Ultrasound for Electromechanical Wave Imaging to Differentiate Endocardial From Epicardial Myocardial Activation
}

Francis Bessière, Ali Zorgani, Jade Robert, Loïc Daunizeau, Elodie Cao, Fanny Vaillant, Emma Abell, B. Quesson, Stéphane Catheline, Philippe Chevalier, et al.

\section{To cite this version:}

Francis Bessière, Ali Zorgani, Jade Robert, Loïc Daunizeau, Elodie Cao, et al.. High Frame Rate Ultrasound for Electromechanical Wave Imaging to Differentiate Endocardial From Epicardial Myocardial Activation. Ultrasound in Medicine \& Biology, 2020, 46 (2), pp.405-414. 10.1016/j.ultrasmedbio.2019.10.017 . hal-03033116

\section{HAL Id: hal-03033116 https://hal.science/hal-03033116}

Submitted on 3 Dec 2020

HAL is a multi-disciplinary open access archive for the deposit and dissemination of scientific research documents, whether they are published or not. The documents may come from teaching and research institutions in France or abroad, or from public or private research centers.
L'archive ouverte pluridisciplinaire HAL, est destinée au dépôt et à la diffusion de documents scientifiques de niveau recherche, publiés ou non, émanant des établissements d'enseignement et de recherche français ou étrangers, des laboratoires publics ou privés. 
$1 \quad$ High frame rate ultrasound for electromechanical wave

2

4

5 Francis Bessière MD MSc ${ }^{1,2,3}$, Ali Zorgani $\mathrm{PhD}^{2,3}$, Jade Robert $\mathrm{MSc}^{2,3}$, Loïc Daunizeau

$6 \mathrm{MSc}^{2,3}$, Elodie Cao $\mathrm{MSc}^{2,3}$, Fanny Vaillant $\mathrm{PhD}^{4,5,6}$, Emma Abell MSc $\mathrm{Mc}^{4,5,6}$, Bruno Quesson

$7 \quad \mathrm{PhD}^{4,5,6}$, Stéphane Catheline $\mathrm{PhD}^{2,3}$, Philippe Chevalier $\mathrm{MD} \mathrm{PhD}^{1,3}$, Cyril Lafon $\mathrm{PhD}^{2,3}$

8

9

None of the authors report any conflict of interest

1- Hôpital cardiologique Louis Pradel, Hospices Civils de Lyon, 69677 Lyon, France

2- LabTAU, INSERM, Centre Léon Bérard, Université Lyon 1, Univ Lyon, F-69003, LYON, France

3- Université de Lyon, France

4- IHU Liryc, Electrophysiology and Heart Modeling Institute, fondation Bordeaux Université, 33600 Pessac-Bordeaux, France

5- Université de Bordeaux, Centre de recherche Cardio-Thoracique de Bordeaux, U1045, 33000, Bordeaux, France

6- INSERM, Centre de recherche Cardio-Thoracique de Bordeaux, U1045, 33000 Bordeaux, France

myocardial activation 
1 Corresponding author:

2 Dr Francis BESSIERE

3 Electrophysiology department, Louis Pradel Cardiovascular Hospital

459 boulevard Pinel, 69500 Bron, France

$5 \quad$ E mail : francis.bessiere@,chu-lyon.fr

6 Tel : +33472119075

7

8

9

10

11

12

13

14

15

16

17

18

19

20

21

22

23

24 
3 Differentiation between epicardial and endocardial ventricular activation remains a challenge,

4 despite the latest technologies available. The aim of the present study was to develop a new

5 tool method, based on electromechanical wave imaging (EWI), to improve arrhythmogenic

6 substrate activation analysis. Experiments were conducted on left ventricles (LV) of four

7 isolated working mode swine hearts. The protocol aimed at demonstrating that different 8 patterns of mechanical activation could be observed whether the ventricle was in sinus

9 rhythm, paced from the epicardium, or from the endocardium. Seventy-two EWI acquisitions

10 were recorded on the anterior, lateral, and posterior segments of the LV. Fifty-four loop

11 records were blindly assigned to two readers. EWI sequences interpretations were correct in

$1289 \%$ of cases. The overall agreement rate between the two readers was $83 \%$. When in a 13 paced ventricle, the origin of the wave front was focal and originated from the endocardium 14 or the epicardium. In sinus rhythm, wave front was global and activated within the entire 15 endocardium towards the epicardium at a speed of $1.7 \pm 0.28 \mathrm{~m} . \mathrm{s}-1$. Wave front speeds were 16 respectively measured when the endocardium or the epicardium were paced at a speed of 1.1 $17 \pm 0.35$ m.s-1 vs $1.3 \pm 0.34$ m.s-1 ( $\mathrm{p}=\mathrm{NS})$. EWI activation mapping allows activation localization within the LV wall and calculation of the wave front propagation speed through the muscle. In the future, this technology could help localize activation within the LV thickness during complex ablation procedures.

23 Keywords: Electromechanical wave imaging; ventricular arrhythmia; endocardial versus epicardial activation; cardiac mapping; ultrasound 
3 Many options are available to delineate the electrophysiological and anatomical substrates of

4 ventricular arrhythmia, whether it concerns premature ventricular contraction (PVC) or 5 ventricular tachycardia (VT). The electrocardiogram (ECG) is the oldest option. QRS 6 complex duration, polarity, and morphology analysis from a 12-lead surface trace provides 7 clues on the location of the arrhythmia inside the ventricles. However, ECG lacks precision 8 since it is difficult to localize the origin of a foci within the thickness of the wall. Such 9 difficulties have already been reported when differentiating endocardial from epicardial VT(Fernandez-Armenta and Berruezo 2014) in the left ventricle (LV), and when attempting

11 to localize PVC in the outflow tract (Lerman 2015)(Enriquez et al. 2017). Recently, high12 density extra-cardiac mapping has been developed. A 252-lead surface acquisition merged onto a three dimensional cardiac tomography reconstruction provides real time activation mapping to localize the arrhythmia source (J Shah et al. 2013)'(Revishvili et al. 2015)'(Erkapic et al. 2015). This promising technology can be used to locate occult paroxysmal arrhythmia during catheter ablation procedures. However, this method is expensive and lacks precision since the body surface acquisition is only able to show the first area activated. In the case of PVC or VT, the system cannot distinguish endocardial from epicardial origin, as the electrical recordings at the surface of the thorax combine information from the entire thickness of the ventricles. A bipolar catheter-based endocardial and epicardial activation mapping system can also be used to more precisely localize the arrhythmia. In such a context, if the underlying electrophysiological mechanism is hyper automatism, it usually allows the localization of a 23 PVC or a VT and when it is a reentrant circuit, the critical isthmus may be determined 24 effectively. However, this technique requires time to yield reliable and useful information to 25 locate and treat the arrhythmia substrate. Sometimes, the exact location of a PVC or VT 
1 remains unclear, since it may come from the mid myocardium, or an area that cannot be

2 reached easily by an intra-cardiac catheter (i.e: LV summit)(Enriquez et al. 2017). Moreover,

3 this method remains risky since epicardial access may lead to coronary artery injuries, cardiac

4 perforation, or chronic pericarditis and endocardial access may cause strokes(Shivkumar 5 2019).

6 Ultrasonic quantitative mapping of myocardial activation has been shown to provide global 7 activation maps. High rate recordings from myocardial deformation are gated by the 8 electrocardiogram in such a way that motion can be decomposed while the ECG runs to create 9 the electromechanical wave imaging (EWI). This has been shown to be effective in mapping 10 sinus rhythm activation(Melki et al. 2017) or stimulated rhythm(Bunting et al. 2017)'(Costet 11 et al. 2016) in animal models with healthy, infarcted(Costet et al. 2017), or ablated 12 hearts(Papadacci et al. 2017a). More recently, EWI was able to differentiate endocardial from epicardial foci (Costet et al. 2018). However, only eight measurements were performed and 14 interpretation was done in a non-blinded manner, preventing a statistically relevant conclusion 15 to be given. In the present preliminary study, experiments were conducted in swine healthy 16 hearts of an isolated working heart model(Vaillant et al. 2016) using local bipolar electrogram 17 signal gated EWI. The aim was to describe local high-resolution endocardial and epicardial activation patterns and characterize wave front propagation within the thickness of the LV wall. Reliability of this method was provided from EWI records of the anterior, lateral, and posterior walls of the LV. 
3 The study protocol was approved by the local Animal Research Ethics Committee (CEEA50)

4 in accordance with recommendations of the Directive 2010/63/EU of the European

5 Parliament on the protection of animals used for scientific purposes. Pigs (Large White

6 Landrace, $40 \mathrm{~kg}, n=4)$ were pre-medicated with an intramuscular injection of ketamine (20

$7 \mathrm{mg} / \mathrm{kg})$, acepromazine $(0.1 \mathrm{mg} / \mathrm{kg})$, and buprenorphine $(10 \mu \mathrm{g} / \mathrm{kg})$. Anesthesia was induced

8 with an intravenous bolus of propofol $(1 \mathrm{mg} / \mathrm{kg})$ and maintained with ketamine and

9 midazolam (40 mg. $\mathrm{kg}^{-1} \cdot \mathrm{h}^{-1}$ and $2 \mathrm{mg} \cdot \mathrm{kg}^{-1} \cdot \mathrm{h}^{-1}$, respectively). Animals were then intubated and

10 ventilated (50/50 air/oxygen), and received an injection of heparin (200 UI/kg).

Excision and perfusion

12 The swine thorax was opened, and blood was collected (2 L) via the introduction of an 8-Fr 13 sheath into the right jugular vein. Heparin $(2500 \mathrm{UI} / \mathrm{L})$ was added in the reservoir to avoid 14 coagulation. Heart was excised according to protocols used in humans during heart 15 transplantation; cardiac arrest was performed in vivo by cross clamping of the ascending aorta 16 and direct injection into the aortic root of $1 \mathrm{~L}$ of cold $\left(4^{\circ} \mathrm{C}\right)$ cardioplegic homemade Custodiol 17 (in mM: $\mathrm{NaCl} 15, \mathrm{KCl} 9, \mathrm{MgCl} 2$ 4, $\alpha$-ketoglutaric acid 1, histidine.HCl.H2O 18, histidine 18 180, tryptophan 2, mannitol $30, \mathrm{CaCl} 20.015$ ), before rapid excision and immersion in a cold $190.9 \%$ saline solution. The aorta, pulmonary artery, pulmonary veins, and superior vena cava 20 were cannulated to perfuse the heart in the isolated working mode. Inferior vena cava vein 21 was sutured to limit leaks. 
1 The chambers used for preload, afterload, and aortic compliance were made from a 1 liter

2 water- jacketed glassware (Radnoti LLC, Monrovia, California, USA) to control the perfusate

3 temperature. Heart reperfusion was performed in the Langendorff mode for 15-20 min to

4 wash out the cardioplegic solution, gradually rewarm the heart and recover a stable ex vivo

5 cardiac function. The Langendorff perfusion pressure was adjusted to achieve $60 \mathrm{mmHg}$. If

6 necessary, the heart was defibrillated at 10-30 J (Life Pak 12, Medtronic, Minneapolis,

7 Minnesota, USA). The heart was perfused with blood collected on the same animal before

8 heart excision, diluted with a Tyrode buffer (vol/vol: 1/5; in mM: $\mathrm{NaCl} 128, \mathrm{KCl} 4.7, \mathrm{MgCl}_{2}$

$9 \quad 0.7, \mathrm{NaH}_{2} \mathrm{PO}_{4}$ 0.5, $\mathrm{NaHCO}_{3} 28, \mathrm{CaCl}_{2}$ 1.8, Glucose 11, Pyruvate 0.5, Lactate 1, Mannitol 16).

10 The perfusion medium was gassed with a mix of $95 \% / 5 \%$ oxygen for maximal oxygenation

11 and maintained at $\mathrm{pH} 7.4$ and at $37.5^{\circ} \mathrm{C}$. A clinical oxygenator and arterial filter (Getinge

12 Group, Rastatt, Bade-Wurtember, Germany) were added before the preload reservoir to ensure an optimal oxygenation of the perfusion medium. After stabilization (10 min with spontaneous cardiac rhythm), the system was switched from Langendorff to working mode.

Pre- and afterloads were maintained constant during the whole protocol (left atrial pressure: 10-15 mmHg; right atrial pressure: 5-10 $\mathrm{mmHg}$; left ventricular afterload: 70-90 mmHg; right ventricular afterload: $15-30 \mathrm{mmHg}$ ).

Lead placement and pacing protocol

Six pacemaker leads (Tendril 52cm, Abbott, Minneapolis, Minnesota, USA) were fixed in pairs, on the anterior, lateral, and posterior walls of the LV. The epicardial leads were attached on the epicardium of the designated portion; under ultrasound guidance endocardial leads were screwed at the exact opposite side of the wall, in such a way that the exact same portion of the LV could be paced from the endocardium or the epicardium. A schematic view 
1 and description of the setup is shown in Figures $1 \& 2$. Each lead was successively connected

2 via a wire to a device controller (Merlin, Abbott, Minneapolis, Minnesota, USA). Pacing 3 parameters were settled $0.2 \mathrm{~V}$ above the threshold to get a local capture that would not

4 jeopardize activation mapping within the LV wall. The duration of the stimulus was set at 0.4 5 ms. Sites were consecutively paced $20 \mathrm{bpm}$ above the spontaneous sinus rhythm.

Ultrasound acquisition

9 A $15 \mathrm{MHz}$ central frequency ultrasonic probe (L15-Xtech, Vermon, Tours, France) was placed facing the LV wall (Figure 2) and maintained by a hydraulic flexible arm in the same

11 position during the whole experiment. The 2-dimensional probe was connected to an ultrafast 12 ultrasound scanner (Vantage, Verasonics, Kirkland, Washington, USA). Conventional Bmode images were acquired in the short-axis at 50 frames/second to assist myocardial segmentation and to guide the placement of the pacing electrodes. After placing two electrodes at the surface of the heart, a bipolar signal was acquired using an ECG unit (DAM 50, Word Precision Instrument, Sarasota, Florida, USA) and recorded using an oscilloscope (PicoScope 3000, Pico Technology, St Neots, UK).

An ultrafast imaging sequence was used to acquire 400 B-mode images at a pulse repetition frequency of 2500 frames/second on the first heart $(160 \mathrm{~ms})$. The last three hearts were recorded using 800 B-mode images acquisition at a pulse repetition frequency of 4000

21 frames/second (200ms), during sinus rhythm, pericardial, or endocardial pacing to maximize

22 the accuracy of the measurements. In order to synchronize the ultrafast acquisition with the 23 bipolar signal wave, an output signal was generated for each frame and superimposed on the 24 bipolar signal. A phase-based motion tracking estimation algorithm(Pinton et al. 2006) was 25 applied to the radiofrequency (RF) data in order to track the electromechanical wave 
1 propagation into the LV wall. Unlike speckle tracking algorithm based on RF-Window cross-

2 correlation, this method allows conservation of high spatial resolution of the initial B-mode 3 images.

EWI interpretation

7 A total of 4 isolated hearts were analyzed successively. The experiments on the first 2 hearts were carried out to validate the proof of concept. Loops from the anterior wall on the left ventricle were analyzed to describe the scheme of activation when the electrical activation was coming from the epicardium, the endocardium, and in sinus rhythm. Based on the observations from the first 2 hearts, the last 2 hearts were carried out to evaluate the ability of the system to detect the different patterns of mechanical activation when blinded to the electrical activation. Three segments of the LV were successively analyzed: the anterior wall, the lateral wall, and the posterior wall. For every segment and activation mode, EWI loops were recorded three times. Two separate readers (JR and EC) received training based of the observations obtained from the first 2 hearts. Then, blinded to the electrical activation, the same authors qualitatively interpreted the 3-recorded loops of a segment to be in accordance with the predetermined sinus rhythm, endocardial pacing, and epicardial pacing patterns. A sequence of a 3-times recorded loop was considered in accordance when at least 2 of the 3 loops were consistent with predetermined patterns. The axial propagation speeds of the wave fronts were computed based on a time of flight method along the thickness of the myocardium wall (i.e. time vs space representation)(Nguyen et al. 2014). Quantitative (mean \pm standard deviation (SD)) and qualitative (\%) variables were analyzed. Overall agreement analyses were performed using the Cohen's Kappa index. 
3 From the first two hearts, 6 EWI sequences were collected and analyzed: 2 in sinus rhythm, 2

4 in epicardial pacing, and 2 in endocardial pacing. EWI sequences were each made by a 35 recordings of the zone (= 18 loops). In sinus rhythm, visual qualitative displays of the wave

6 front scheme were similar between all recordings: the EWI-induced wave front systematically 7 originated from the endocardium and mid myocardium and moved towards the epicardium 8 with a wide and global displacement (Figure 3). During epicardial and endocardial pacing, 9 visual qualitative displays of the wave front demonstrated a focal positive displacement after 10 rising of the local electrical signal. When visible, the origin of the EWI activation matched the 11 location of the epicardial or endocardial leads (Figures 4 and 5).

13 From the last two hearts, 18 EWI sequences were collected: 6 in sinus rhythm, 6 in epicardial pacing, and 6 in endocardial pacing. EWI sequences were each made by a 3-recordings of the zone (=54 loops). Every sequence in sinus rhythm, epicardial pacing, and endocardial pacing were all collected 2 times on the anterior, lateral wall, and posterior walls. Two independent readers analyzed all sequences to provide descriptive statistics $(36$ lectures $=108$ loops analyzed).

In spontaneous sinus rhythm, the mean $\pm \mathrm{SD}$ heart rate was $119 \pm 6 \mathrm{bpm}$; the epicardium and endocardium sides were sequentially paced at a mean \pm SD rate of $138 \pm 9 \mathrm{bpm}$.

22 EWI sequences readings matched the electrical activation in $89 \%$ of cases (Table 1). The overall agreement rate between the two readers was $83 \%$. 
1 The readers differentiated in $100 \%$ of cases, paced from sinus rhythm activations, on the

2 anterior, the lateral, and the posterior walls. Mean \pm SD propagation speed of the mechanical

3 wave front in sinus rhythm, into the thickness of the muscle, was $1.7 \pm 0.28 \mathrm{~m} . \mathrm{s}^{-1}$.

4 EWI acquisitions allowed epicardial activation detection in $92 \%$ of cases. When on the

5 posterior or anterior walls, activation patterns from the epicardium were analyzed correctly in

$6100 \%$ of cases. When on the lateral wall, only $75 \%$ of correct analysis was observed (Table

7 1). The mechanical wave front started in a focal zone where the lead was located, and 8 propagated from the epicardium towards the endocardium at a mean $\pm \mathrm{SD}$ speed of $1.3 \pm 0.34$

$9 \mathrm{~m} . \mathrm{s}^{-1}$. EWI acquisitions allowed endocardial activation detection in $75 \%$ of cases. When on 10 the posterior wall, activation patterns detected from the endocardial lead were analyzed 11 correctly in $100 \%$ of cases against $75 \%$ of correct analyses from the lateral wall and $50 \%$ 12 from the anterior wall (Table 1). The mechanical wave front clearly propagated from the endocardium towards the epicardium at a mean \pm SD speed of $1.1 \pm 0.35 \mathrm{~m} \cdot \mathrm{s}^{-1}$. 


\section{Discussion}

2 EWI mapping allows accurate description of a focal ventricular activation

3 Assessment of the activation through electromechanical wave front motion was effective:

4 endocardial versus epicardial stimulation were clearly distinguished using the location of the

5 initial activation and the direction of the wave front propagation. Sinus rhythm was

6 distinguished from paced rhythm using the shape of the wave front and the electromechanical

7 coupling. The present experiment was useful not only to visualize the origin of the

8 mechanical activation within the thickness of a cardiac wall, but also to provide data on the

9 behavior of the wave front into the muscle. Interferences that could jeopardize proper

10 interpretation were distinguished from the electromechanical activation by using the 3-times

11 recorded loops method. Although such a description is still in experimental phase, EWI

12 mapping is promising since the localization of intra myocardial foci may be critical during

13 catheter ablation procedures. It may provide real-time mechanistic insights by linking the

14 wave front speed to histological characteristics of the substrate and be complementary to

15 electro-anatomical mapping systems already available. This technique, more accurate, might

16 help in differentiating the origin of a VT (endocardial versus epicardial) and the underlying 17 mechanism (focal versus reentrance). So far, not a single reliable system enables such 18 observations.

Technical concerns and observations

20 In order to place pacemaker leads easily, at the endocardial and epicardial sides of the three

21 LV portions, experiments were conducted on an isolated working heart instead of a standard

22 in vivo setup. When placing the US probe against the heart, such installation avoids any

23 acoustic fences that can jeopardize the high-resolution activation recordings using an extra 
1 cardiac probe. Since the recordings were performed under a time frame of $200 \mathrm{~ms}$ and the

2 initiation of the EWI was visible during less than $50 \mathrm{~ms}$, global heart motion did not

3 jeopardize visualization. Even though the $15 \mathrm{MHz}$ probe used prevented a large visual field

4 (only $14 \mathrm{~mm}$ x $14 \mathrm{~mm}$ square), it offered an accurate view of the muscle. Konofagou's group

5 recently demonstrated a correlation between EWI activation and global electrical activation in

6 a ventricle(Grondin et al. 2016)'(Costet et al. 2016)'(Provost et al. 2011). Their method

7 provides global activation mapping during sinus rhythm(Melki et al. 2017) or stimulated

8 rhythm(Bunting et al. 2017)'(Costet et al. 2016). More recently, epicardial and endocardial

9 pacing were differentiated in $86 \%$ of cases(Costet et al. 2018) but results were based on only

10 eight measurements and did not take into account activation in sinus rhythm. The experiment

11 presented herein confirmed the possibility to differentiate between endocardial and epicardial

12 focal activation with similar rates and adds a complete analysis of the different segments of 13 the LV. Moreover, it allowed measuring of the wave front speed.

14 Finally, the loop-recorded images were confined to a small area but this technique will 15 eventually be able to provide data about a region of interest, once a 3-dimensionnal electro16 anatomical mapping is performed.

Is there a correlation between myocardial structure and EWI?

20 Different activation wave speeds were observed. The large difference between mechanical 21 and electrical waves reported to be around $0.5 \mathrm{~m} \cdot \mathrm{s}^{-1}$ in human hearts(Durrer et al. 1970) points 22 to the strong possibility that a mechanical wave - once initiated by the "kick" of an electrical 23 stimulus - mainly depends on the mechanical properties of the tissue. Wave front speed 24 changes could be related to the orientation of the myocardial fibers that follow different 
1 directions before reaching the Purkinje system and may help to determine new properties of

2 the cardiac muscle.

3 Even though spatial resolution of the sequence presented herein prevents high-resolution

4 analysis of the activated wave front shape and speed, this technique might support the in vivo 5 correlation of anisotropic properties with the orientations of epicardial and endocardial 6 fibers(Deng et al. 2012), the anatomy of the Purkinje that usually contains more denser fibers 7 close to the endocardium(Garcia-Bustos et al. 2017), and the dispersion of the gap junction 8 density from the endocardium towards the epicardium(Yamada et al. 2004). Further 9 evaluations will be needed, combining information from a 3-dimensional histological analysis 10 of the interlaced fibers and a 3-dimensional ultrasound analysis of the area.

\section{Limitations}

The present study has some limitations. All images were obtained in swine hearts, in an isolated bi-ventricular working mode with a 2-D probe. Results were therefore based on mechanical recordings from a 2 dimensional space of an artificial endocardial and epicardial paced activation. Moreover, since the hearts were healthy, no pathological substrate was present inside the muscle. Even though 2-D spatial analyses sometimes prevented the wave front to be clearly visible, the propagation of the electromechanical waves was looking homogenous making the identification of the paced site easier and the calculation of the mechanical wave front speed possible. Interestingly, global EWI mapping has already been performed in sinus rhythm of myocardial infarction models(Costet et al. 2017), and on ablated

23 myocardium(Papadacci et al. 2017a). Since substrate analysis was successfully achieved in 24 those models, clinical application should be attainable. However, VT would induce more 25 complex schemes of activation in the three dimensions. Future experiments are needed using 
1 a three-dimensional ultrasound and pathological models to determine the local EWI into a

2 scar and the ability of the system to determine a reentrant circuit.

3

$4 \quad$ Ultrasound based mapping and its part in the future of electrophysiology

6 Since the understanding of underlying mechanisms during VT ablation is one of the 7 cornerstones of a successful procedure, the type of acquisition presented here could be 8 integrated with electro-anatomical data, during catheter-based procedure, in order to improve 9 ablation success. This sequence could be embedded into an intra-cardiac echography (ICE) 10 system, avoiding any acoustic fences that could compromise quality of the images. Since ICE 11 already offers 2-D images and should be providing 3-D images in the next decade, the 12 addition of such sequences should allow a real-time "single beat" EWI activation map of 13 interlaced myocardial fibers inside an arrhythmic ventricle wall(Papadacci et al. 2017b). 14 Another option to limit acoustic fences would consist in providing such sequences under 15 transesophageal echocardiography (TEE) analysis, which already offers 2-D and 3-D 16 acquisitions. The combination of EWI mapping with elastography (available with ICE and 17 TEE) could provide both the location of the arrhythmia and the characteristics (shape, surface, volume) of the thermal lesion after the ablation(Kwiecinski et al. 2015)'(Kwiecinski et al. 2014)'(Sandoval et al. 2018). Since high-intensity focused ultrasounds can be delivered from a TEE(Bessiere et al. 2016)'(Constanciel et al. 2013) into the ventricles, ultrasound energy 21 might be able, in the future, to localize, treat, and monitor cardiac arrhythmias.

\section{Conclusions}


1 EWI mapping allows assessment of mechanical activation of a LV wall in healthy hearts of an

2 isolated working mode system. Endocardial and epicardial pacing can be easily differentiated

3 from sinus rhythm activation and EWI-induced wave front can be accurately analyzed. This

4 technique is promising to provide help in differentiating between endocardial and epicardial

5 arrhythmias, during complex ablation procedures, and to characterize the mechanism of

6 ventricular activation. Future experiments will need to be conducted in diseased hearts, and

7 clinical data will be needed to correlate electrophysiological data to EWI activation patterns.

8

9

\section{Acknowledgements}

17 This work was supported by the French National Research Agency (ANR) under the

18 CHORUS Grant project ANR17-CE19-0017 and France Life Imaging - WP3 2019. Dr

19 Verena Landel is warmly thanked for her contribution and proofreading of the manuscript. 
3 Bessiere F, N'djin WA, Colas EC, Chavrier F, Greillier P, Chapelon JY, Chevalier P, Lafon

4 C. Ultrasound-Guided Transesophageal High-Intensity Focused Ultrasound Cardiac Ablation 5 in a Beating Heart: A Pilot Feasibility Study in Pigs. Ultrasound Med Biol 2016;42:184861861.

7 Bunting E, Lambrakos L, Kemper P, Whang W, Garan H, Konofagou E. Imaging the 8 Propagation of the Electromechanical Wave in Heart Failure Patients with Cardiac 9 Resynchronization Therapy. Pacing Clin Electrophysiol PACE 2017;40:35-45.

Constanciel E, N'Djin WA, Bessière F, Chavrier F, Grinberg D, Vignot A, Chevalier P,

11 Chapelon JY, Lafon C. Design and evaluation of a transesophageal HIFU probe for 12 ultrasound-guided cardiac ablation: simulation of a HIFU mini-maze procedure and preliminary ex vivo trials. IEEE Trans Ultrason Ferroelectr Freq Control 2013;60:1868-1883.

Konofagou E. Electromechanical wave imaging and electromechanical wave velocity estimation in a large animal model of myocardial infarction. Phys Med Biol 2017;62:93419356.

Costet A, Wan E, Bunting E, Grondin J, Garan H, Konofagou E. Electromechanical wave imaging (EWI) validation in all four cardiac chambers with 3D electroanatomic mapping in canines in vivo. Phys Med Biol 2016;61:8105-8119.

Costet A, Wan E, Melki L, Bunting E, Grondin J, Garan H, Konofagou E. Non-invasive Characterization of Focal Arrhythmia with Electromechanical Wave Imaging in Vivo.

23 Ultrasound Med Biol 2018;

24 Deng D, Jiao P, Ye X, Xia L. An image-based model of the whole human heart with detailed 25 anatomical structure and fiber orientation. Comput Math Methods Med 2012;2012:891070. 
1 Durrer D, van Dam RT, Freud GE, Janse MJ, Meijler FL, Arzbaecher RC. Total excitation of

2 the isolated human heart. Circulation 1970;41:899-912.

3 Enriquez A, Malavassi F, Saenz LC, Supple G, Santangeli P, Marchlinski FE, Garcia FC.

4 How to map and ablate left ventricular summit arrhythmias. Heart Rhythm 2017;14:141-148.

5 Erkapic D, Greiss H, Pajitnev D, Zaltsberg S, Deubner N, Berkowitsch A, Möllman S,

6 Sperzel J, Rolf A, Schmitt J, Hamm CW, Kuniss M, Neumann T. Clinical impact of a novel

7 three-dimensional electrocardiographic imaging for non-invasive mapping of ventricular

8 arrhythmias-a prospective randomized trial. Eur Eur Pacing Arrhythm Card Electrophysiol J

9 Work Groups Card Pacing Arrhythm Card Cell Electrophysiol Eur Soc Cardiol 2015;17:59110597.

11 Fernandez-Armenta J, Berruezo A. How to recognize epicardial origin of ventricular 12 tachycardias? Curr Cardiol Rev 2014;10:246-256.

13 Garcia-Bustos V, Sebastian R, Izquierdo M, Molina P, Chorro FJ, Ruiz-Sauri A. A

14 quantitative structural and morphometric analysis of the Purkinje network and the Purkinje15 myocardial junctions in pig hearts. J Anat 2017;230:664-678.

16 Grondin J, Costet A, Bunting E, Gambhir A, Garan H, Wan E, Konofagou EE. Validation of 17 Electromechanical Wave Imaging in a canine model during pacing and sinus rhythm. Heart 18 Rhythm Off J Heart Rhythm Soc 2016;13:2221-2227.

19 J Shah A, Hocini M, Pascale P, Roten L, Komatsu Y, Daly M, Ramoul K, Denis A, Derval N,

20 Sacher F, Dubois R, Bokan R, Eliatou S, Strom M, Ramanathan C, Jais P, Ritter P,

21 Haissaguerre M. Body Surface Electrocardiographic Mapping for Non-invasive Identification

22 of Arrhythmic Sources. Arrhythmia Electrophysiol Rev 2013;2:16-22.

23 Kwiecinski W, Bessière F, Colas EC, N'Djin WA, Tanter M, Lafon C, Pernot M. Cardiac 24 shear-wave elastography using a transesophageal transducer: application to the mapping of 25 thermal lesions in ultrasound transesophageal cardiac ablation. Phys Med Biol 2015;60:7829- 
2 Kwiecinski W, Provost J, Dubois R, Sacher F, Haïssaguerre M, Legros M, Nguyen-Dinh A,

3 Dufait R, Tanter M, Pernot M. Quantitative evaluation of atrial radio frequency ablation using

$4 \quad$ intracardiac shear-wave elastography. Med Phys 2014;41:112901.

5 Lerman BB. Mechanism, diagnosis, and treatment of outflow tract tachycardia. Nat Rev

6 Cardiol 2015;12:597-608.

7 Melki L, Costet A, Konofagou EE. Reproducibility and Angle Independence of

8 Electromechanical Wave Imaging for the Measurement of Electromechanical Activation

9 during Sinus Rhythm in Healthy Humans. Ultrasound Med Biol 2017;43:2256-2268.

10 Nguyen T-M, Song S, Arnal B, Huang Z, O’Donnell M, Wang RK. Visualizing ultrasonically

11 induced shear wave propagation using phase-sensitive optical coherence tomography for

12 dynamic elastography. Opt Lett 2014;39:838-841.

13 Papadacci C, Bunting EA, Wan EY, Nauleau P, Konofagou EE. 3D myocardial elastography

14 and electromechanical wave imaging in vivo. IEEE Trans Med Imaging 2017a;36:618-627.

15 Papadacci C, Finel V, Provost J, Villemain O, Bruneval P, Gennisson J-L, Tanter M, Fink M,

16 Pernot M. Imaging the dynamics of cardiac fiber orientation in vivo using 3D Ultrasound

17 Backscatter Tensor Imaging. Sci Rep 2017b;7:830.

18 Pinton GF, Dahl JJ, Trahey GE. Rapid tracking of small displacements with ultrasound. IEEE

19 Trans Ultrason Ferroelectr Freq Control 2006;53:1103-1117.

20 Provost J, Lee W-N, Fujikura K, Konofagou EE. Imaging the electromechanical activity of

21 the heart in vivo. Proc Natl Acad Sci U S A 2011;108:8565-8570.

22 Revishvili AS, Wissner E, Lebedev DS, Lemes C, Deiss S, Metzner A, Kalinin VV, Sopov

23 OV, Labartkava EZ, Kalinin AV, Chmelevsky M, Zubarev SV, Chaykovskaya MK, Tsiklauri

24 MG, Kuck K-H. Validation of the mapping accuracy of a novel non-invasive epicardial and

25 endocardial electrophysiology system. Eur Eur Pacing Arrhythm Card Electrophysiol J Work 
1 Groups Card Pacing Arrhythm Card Cell Electrophysiol Eur Soc Cardiol 2015;17:1282-1288.

2 Sandoval Z, Castro M, Alirezaie J, Bessière F, Lafon C, Dillenseger J-L. Transesophageal 2D

3 ultrasound to 3D computed tomography registration for the guidance of a cardiac arrhythmia

$4 \quad$ therapy. Phys Med Biol 2018;63:155007.

5 Shivkumar K. Catheter Ablation of Ventricular Arrhythmias. N Engl J Med 2019;380:1555-

61564.

7 Vaillant F, Magat J, Bour P, Naulin J, Benoist D, Loyer V, Vieillot D, Labrousse L, Ritter P,

8 Bernus O, Dos Santos P, Quesson B. Magnetic resonance-compatible model of isolated

9 working heart from large animal for multimodal assessment of cardiac function,

10 electrophysiology, and metabolism. Am J Physiol Heart Circ Physiol 2016;310:H1371-1380.

11 Yamada KA, Kanter EM, Green KG, Saffitz JE. Transmural distribution of connexins in

12 rodent hearts. J Cardiovasc Electrophysiol 2004;15:710-715.

13

14

15

16

17

18

19

20

21

22

23

24 
1 Table 1 Summary of the correct Electromechanical Wave Imaging (EWI) sequences

2 interpretations from two independent readers

\begin{tabular}{|c|c|c|c|c|c|c|}
\hline \multicolumn{3}{|c|}{ Activation origin } & \multirow{2}{*}{$\begin{array}{c}\text { EPICARDIUM } \\
\text { 3/4 }\end{array}$} & \multirow{2}{*}{$\begin{array}{l}\text { ENDOCARDIUM } \\
\qquad 3 / 4\end{array}$} & \multirow{2}{*}{$\begin{array}{c}\text { SINUS } \\
\text { RHYTHM } \\
4 / 4\end{array}$} & \multirow{2}{*}{$\begin{array}{l}\text { TOTAL } \\
10 / / 12\end{array}$} \\
\hline \multirow{8}{*}{ SEGMENT } & \multirow{2}{*}{ LATERAL } & $\mathrm{n}$ & & & & \\
\hline & & $\%$ & $75 \%$ & $75 \%$ & $100 \%$ & $83 \%$ \\
\hline & \multirow{2}{*}{ POSTERIOR } & $\mathrm{n}$ & $4 / 4$ & $4 / 4$ & $4 / 4$ & $12 / 12$ \\
\hline & & $\%$ & $100 \%$ & $100 \%$ & $100 \%$ & $100 \%$ \\
\hline & \multirow{2}{*}{ ANTERIOR } & $\mathrm{n}$ & $4 / 4$ & $2 / 4$ & $4 / 4$ & $10 / 12$ \\
\hline & & $\%$ & $100 \%$ & $50 \%$ & $100 \%$ & $83 \%$ \\
\hline & \multirow{2}{*}{ TOTAL } & $\mathrm{n}$ & $11 / 12$ & $9 / 12$ & $12 / 12$ & $32 / 36$ \\
\hline & & $\%$ & $92 \%$ & $75 \%$ & $100 \%$ & $89 \%$ \\
\hline
\end{tabular}

3

4

5

6

7

8

9

10

11

12

13

14

15

16

17 


\section{Figure Captions}

2

3 Figure 1: Isolated working mode heart. The $15 \mathrm{MHz}$ ultrasound (US) probe is facing the left

4 ventricle (LV). Two leads are screwed into the epicardial and endocardial sides of the LV.

5 The one on the endocardial side is placed through the left atrium, from a pulmonary vein.

6

7 Figure 2: The cross-cut view of the left ventricle depicts the relation between the epicardial 8 and endocardial leads and the ultrasound probe (short-axis).

9 the epicardial lead.

Figure 3: Electromechanical wave imaging (EWI) in sinus rhythm. The first column shows the electrocardiogram: from the first to the last line, a red dot depicts when the images were recorded at four different moments of a cardiac cycle (ms). The second column shows the Bmode corresponding images. The third column shows the corresponding EWI pictures (deformation depicted in $\mu \mathrm{m}$ ). The beginning of the induced electromechanical wave front is wide, originating from the endocardium towards the epicardium.

Figure 4: EWI during epicardial pacing. The first column shows the electrocardiogram: from the first to the last line, a red dot depicts when the images were recorded at four different moments of a cardiac cycle (ms). The second column shows the B-mode corresponding images. The third column shows the corresponding EWI pictures (deformation depicted in $\mu \mathrm{m})$. The beginning of the induced electromechanical wave front is focal and clearly distinguished on the upper right corner of the image (in red). It corresponds to the location of 
1 Figure 5: EWI during endocardial pacing. The first column shows the electrocardiogram:

2 from the first to the last line, a red dot depicts when the images were recorded at four different 3 moments of a cardiac cycle (ms). The second column shows the B-mode corresponding 4 images. The third column shows the corresponding EWI pictures (deformation depicted in $5 \mu \mathrm{m})$. The beginning of the induced electromechanical wave front is focal and clearly 6 distinguished on the lower left corner of the image (in red). It corresponds to the location of 7 the endocardial lead.

8 
$1 \quad$ High frame rate ultrasound for electromechanical wave

2

4

5 Francis Bessière MD MSc ${ }^{1,2,3}$, Ali Zorgani $\mathrm{PhD}^{2,3}$, Jade Robert $\mathrm{MSc}^{2,3}$, Loïc Daunizeau

$6 \mathrm{MSc}^{2,3}$, Elodie Cao $\mathrm{MSc}^{2,3}$, Fanny Vaillant $\mathrm{PhD}^{4,5,6}$, Emma Abell MSc $\mathrm{Mc}^{4,5,6}$, Bruno Quesson

$7 \quad \mathrm{PhD}^{4,5,6}$, Stéphane Catheline $\mathrm{PhD}^{2,3}$, Philippe Chevalier $\mathrm{MD} \mathrm{PhD}^{1,3}$, Cyril Lafon $\mathrm{PhD}^{2,3}$

8

9

None of the authors report any conflict of interest

1- Hôpital cardiologique Louis Pradel, Hospices Civils de Lyon, 69677 Lyon, France

2- LabTAU, INSERM, Centre Léon Bérard, Université Lyon 1, Univ Lyon, F-69003, LYON, France

3- Université de Lyon, France

4- IHU Liryc, Electrophysiology and Heart Modeling Institute, fondation Bordeaux Université, 33600 Pessac-Bordeaux, France

5- Université de Bordeaux, Centre de recherche Cardio-Thoracique de Bordeaux, U1045, 33000, Bordeaux, France

6- INSERM, Centre de recherche Cardio-Thoracique de Bordeaux, U1045, 33000 Bordeaux, France

myocardial activation 
1 Corresponding author:

2 Dr Francis BESSIERE

3 Electrophysiology department, Louis Pradel Cardiovascular Hospital

459 boulevard Pinel, 69500 Bron, France

$5 \quad$ E mail : francis.bessiere@,chu-lyon.fr

6 Tel : +33472119075

7

8

9

10

11

12

13

14

15

16

17

18

19

20

21

22

23

24 
3 Differentiation between epicardial and endocardial ventricular activation remains a challenge,

4 despite the latest technologies available. The aim of the present study was to develop a new

5 tool method, based on electromechanical wave imaging (EWI), to improve arrhythmogenic

6 substrate activation analysis. Experiments were conducted on left ventricles (LV) of four

7 isolated working mode swine hearts. The protocol aimed at demonstrating that different 8 patterns of mechanical activation could be observed whether the ventricle was in sinus 9 rhythm, paced from the epicardium, or from the endocardium. Seventy-two EWI acquisitions 10 were recorded on the anterior, lateral, and posterior segments of the LV. Fifty-four loop 11 records were blindly assigned to two readers. EWI sequences interpretations were correct in $1289 \%$ of cases. The overall agreement rate between the two readers was $83 \%$. When in a 13 paced ventricle, the origin of the wave front was focal and originated from the endocardium 14 or the epicardium. In sinus rhythm, wave front was global and activated within the entire 15 endocardium towards the epicardium at a speed of $1.7 \pm 0.28 \mathrm{~m} . \mathrm{s}-1$. Wave front speeds were 16 respectively measured when the endocardium or the epicardium were paced at a speed of 1.1 $17 \pm 0.35$ m.s-1 vs $1.3 \pm 0.34$ m.s-1 ( $\mathrm{p}=\mathrm{NS})$. EWI activation mapping allows activation localization within the LV wall and calculation of the wave front propagation speed through the muscle. In the future, this technology could help localize activation within the LV 20 thickness during complex ablation procedures.

23 Keywords: Electromechanical wave imaging; ventricular arrhythmia; endocardial versus 24 epicardial activation; cardiac mapping; ultrasound 
3 Many options are available to delineate the electrophysiological and anatomical substrates of

4 ventricular arrhythmia, whether it concerns premature ventricular contraction (PVC) or

5 ventricular tachycardia (VT). The electrocardiogram (ECG) is the oldest option. QRS

6 complex duration, polarity, and morphology analysis from a 12-lead surface trace provides

7 clues on the location of the arrhythmia inside the ventricles. However, ECG lacks precision

8 since it is difficult to localize the origin of a foci within the thickness of the wall. Such

9 difficulties have already been reported when differentiating endocardial from epicardial

10 VT(Fernandez-Armenta and Berruezo 2014) in the left ventricle (LV), and when attempting

11 to localize PVC in the outflow tract (Lerman 2015)(Enriquez et al. 2017). Recently, high-

12 density extra-cardiac mapping has been developed. A 252-lead surface acquisition merged 13 onto a three dimensional cardiac tomography reconstruction provides real time activation 14 mapping to localize the arrhythmia source (J Shah et al. 2013)'(Revishvili et al. 2015)'(Erkapic 15 et al. 2015). This promising technology can be used to locate occult paroxysmal arrhythmia 16 during catheter ablation procedures. However, this method is expensive and lacks precision 17 since the body surface acquisition is only able to show the first area activated. In the case of $\underline{\text { PVC or VT, the system cannot distinguish endocardial from epicardial origin, as the electrical }}$ recordings at the surface of the thorax combine information from the entire thickness of the ventricles. A bipolar catheter-based endocardial and epicardial activation mapping system can

21 also be used to more precisely localize the arrhythmia. In such a context, if the underlying 22 electrophysiological mechanism is hyper automatism, it usually allows the localization of a 23 PVC or a VT and when it is a reentrant circuit, the critical isthmus may be determined 24 effectively. However, this technique requires time to yield reliable and useful information to 25 locate and treat the arrhythmia substrate. Sometimes, the exact location of a PVC or VT 
1 remains unclear, since it may come from the mid myocardium, or an area that cannot be

2 reached easily by an intra-cardiac catheter (i.e: LV summit)(Enriquez et al. 2017). Moreover,

3 this method remains risky since epicardial access may lead to coronary artery injuries, cardiac

4 perforation, or chronic pericarditis and endocardial access may cause strokes(Shivkumar $5 \quad \underline{2019)}$.

6 Ultrasonic quantitative mapping of myocardial activation has been shown to provide global

7 activation maps. High rate recordings from myocardial deformation are gated by the

8 electrocardiogram in such a way that motion can be decomposed while the ECG runs to create

9 the electromechanical wave imaging (EWI). This has been shown to be effective in mapping 10 sinus rhythm activation(Melki et al. 2017) or stimulated rhythm(Bunting et al. 2017)'(Costet 11 et al. 2016) in animal models with healthy, infarcted(Costet et al. 2017), or ablated 12 hearts(Papadacci et al. 2017a). More recently, EWI was able to differentiate endocardial from 13 epicardial foci (Costet et al. 2018). However, only eight measurements were performed and 14 interpretation was done in a non-blinded manner, preventing a statistically relevant conclusion 15 to be given. In the present preliminary study, experiments were conducted in swine healthy 16 hearts of an isolated working heart model(Vaillant et al. 2016) using local bipolar electrogram 17 signal gated EWI. The aim was to describe local high-resolution endocardial and epicardial 18 activation patterns and characterize wave front propagation within the thickness of the LV 19 wall. Reliability of this method was provided from EWI records of the anterior, lateral, and 20 posterior walls of the LV. 
3 The study protocol was approved by the local Animal Research Ethics Committee (CEEA50)

4 in accordance with recommendations of the Directive 2010/63/EU of the European

5 Parliament on the protection of animals used for scientific purposes. Pigs (Large White

6 Landrace, $40 \mathrm{~kg}, n=4)$ were pre-medicated with an intramuscular injection of ketamine (20

$7 \mathrm{mg} / \mathrm{kg})$, acepromazine $(0.1 \mathrm{mg} / \mathrm{kg})$, and buprenorphine $(10 \mu \mathrm{g} / \mathrm{kg})$. Anesthesia was induced

8 with an intravenous bolus of propofol $(1 \mathrm{mg} / \mathrm{kg})$ and maintained with ketamine and

9 midazolam (40 mg. $\mathrm{kg}^{-1} \cdot \mathrm{h}^{-1}$ and $2 \mathrm{mg} \cdot \mathrm{kg}^{-1} \cdot \mathrm{h}^{-1}$, respectively). Animals were then intubated and

10 ventilated (50/50 air/oxygen), and received an injection of heparin (200 UI/kg).

Excision and perfusion

The swine thorax was opened, and blood was collected $(2 \mathrm{~L})$ via the introduction of an $8-\mathrm{Fr}$ sheath into the right jugular vein. Heparin $(2500 \mathrm{UI} / \mathrm{L})$ was added in the reservoir to avoid coagulation. Heart was excised according to protocols used in humans during heart transplantation; cardiac arrest was performed in vivo by cross clamping of the ascending aorta and direct injection into the aortic root of $1 \mathrm{~L}$ of cold $\left(4^{\circ} \mathrm{C}\right)$ cardioplegic homemade Custodiol (in $\mathrm{mM}$ : $\mathrm{NaCl} 15, \mathrm{KCl}$ 9, $\mathrm{MgCl} 2$ 4, $\alpha$-ketoglutaric acid 1, histidine.HCl.H2O 18, histidine 180 , tryptophan 2 , mannitol $30, \mathrm{CaCl} 20.015)$, before rapid excision and immersion in a cold $0.9 \%$ saline solution. The aorta, pulmonary artery, pulmonary veins, and superior vena cava

20 were cannulated to perfuse the heart in the isolated working mode. Inferior vena cava vein 21 was sutured to limit leaks. 
1 The chambers used for preload, afterload, and aortic compliance were made from a 1 liter

2 water- jacketed glassware (Radnoti LLC, Monrovia, California, USA) to control the perfusate

3 temperature. Heart reperfusion was performed in the Langendorff mode for 15-20 min to

4 wash out the cardioplegic solution, gradually rewarm the heart and recover a stable ex vivo

5 cardiac function. The Langendorff perfusion pressure was adjusted to achieve $60 \mathrm{mmHg}$. If

6 necessary, the heart was defibrillated at 10-30 J (Life Pak 12, Medtronic, Minneapolis,

7 Minnesota, USA). The heart was perfused with blood collected on the same animal before

8 heart excision, diluted with a Tyrode buffer (vol/vol: 1/5; in mM: $\mathrm{NaCl} 128, \mathrm{KCl} 4.7, \mathrm{MgCl}_{2}$

$9 \quad 0.7, \mathrm{NaH}_{2} \mathrm{PO}_{4}$ 0.5, $\mathrm{NaHCO}_{3} 28, \mathrm{CaCl}_{2}$ 1.8, Glucose 11, Pyruvate 0.5, Lactate 1, Mannitol 16).

10 The perfusion medium was gassed with a mix of $95 \% / 5 \%$ oxygen for maximal oxygenation

11 and maintained at $\mathrm{pH} 7.4$ and at $37.5^{\circ} \mathrm{C}$. A clinical oxygenator and arterial filter (Getinge

12 Group, Rastatt, Bade-Wurtember, Germany) were added before the preload reservoir to ensure an optimal oxygenation of the perfusion medium. After stabilization (10 min with spontaneous cardiac rhythm), the system was switched from Langendorff to working mode.

Pre- and afterloads were maintained constant during the whole protocol (left atrial pressure: 10-15 mmHg; right atrial pressure: 5-10 $\mathrm{mmHg}$; left ventricular afterload: 70-90 mmHg; right ventricular afterload: $15-30 \mathrm{mmHg}$ ).

Lead placement and pacing protocol

Six pacemaker leads (Tendril 52cm, Abbott, Minneapolis, Minnesota, USA) were fixed in pairs, on the anterior, lateral, and posterior walls of the LV. The epicardial leads were attached on the epicardium of the designated portion; under ultrasound guidance endocardial leads were screwed at the exact opposite side of the wall, in such a way that the exact same portion of the LV could be paced from the endocardium or the epicardium. A schematic view 
1 and description of the setup is shown in Figures $1 \& 2$. Each lead was successively connected

2 via a wire to a device controller (Merlin, Abbott, Minneapolis, Minnesota, USA). Pacing 3 parameters were settled $0.2 \mathrm{~V}$ above the threshold to get a local capture that would not

4 jeopardize activation mapping within the LV wall. The duration of the stimulus was set at 0.4 5 ms. Sites were consecutively paced $20 \mathrm{bpm}$ above the spontaneous sinus rhythm.

Ultrasound acquisition

9 A $15 \mathrm{MHz}$ central frequency ultrasonic probe (L15-Xtech, Vermon, Tours, France) was placed facing the LV wall (Figure 2) and maintained by a hydraulic flexible arm in the same

11 position during the whole experiment. The 2-dimensional probe was connected to an ultrafast 12 ultrasound scanner (Vantage, Verasonics, Kirkland, Washington, USA). Conventional Bmode images were acquired in the short-axis at 50 frames/second to assist myocardial segmentation and to guide the placement of the pacing electrodes. After placing two electrodes at the surface of the heart, a bipolar signal was acquired using an ECG unit (DAM 50, Word Precision Instrument, Sarasota, Florida, USA) and recorded using an oscilloscope (PicoScope 3000, Pico Technology, St Neots, UK).

An ultrafast imaging sequence was used to acquire 400 B-mode images at a pulse repetition frequency of 2500 frames/second on the first heart $(160 \mathrm{~ms})$. The last three hearts were recorded using 800 B-mode images acquisition at a pulse repetition frequency of 4000

21 frames/second $(200 \mathrm{~ms})$, during sinus rhythm, pericardial, or endocardial pacing to maximize

22 the accuracy of the measurements. In order to synchronize the ultrafast acquisition with the 23 bipolar signal wave, an output signal was generated for each frame and superimposed on the 24 bipolar signal. A phase-based motion tracking estimation algorithm(Pinton et al. 2006) was 25 applied to the radiofrequency (RF) data in order to track the electromechanical wave 
1 propagation into the LV wall. Unlike speckle tracking algorithm based on RF-Window cross-

2 correlation, this method allows conservation of high spatial resolution of the initial B-mode 3 images.

EWI interpretation

7 A total of 4 isolated hearts were analyzed successively. The experiments on the first 2 hearts were carried out to validate the proof of concept. Loops from the anterior wall on the left ventricle were analyzed to describe the scheme of activation when the electrical activation was coming from the epicardium, the endocardium, and in sinus rhythm. Based on the observations from the first 2 hearts, the last 2 hearts were carried out to evaluate the ability of the system to detect the different patterns of mechanical activation when blinded to the electrical activation. Three segments of the LV were successively analyzed: the anterior wall, the lateral wall, and the posterior wall. For every segment and activation mode, EWI loops were recorded three times. Two separate readers (JR and EC) received training based of the observations obtained from the first 2 hearts. Then, blinded to the electrical activation, the same authors qualitatively interpreted the 3-recorded loops of a segment to be in accordance with the predetermined sinus rhythm, endocardial pacing, and epicardial pacing patterns. A sequence of a 3-times recorded loop was considered in accordance when at least 2 of the 3 loops were consistent with predetermined patterns. The axial propagation speeds of the wave fronts were computed based on a time of flight method along the thickness of the myocardium wall (i.e. time vs space representation)(Nguyen et al. 2014). Quantitative (mean \pm standard deviation (SD)) and qualitative (\%) variables were analyzed. Overall agreement analyses were performed using the Cohen's Kappa index. 
3 From the first two hearts, 6 EWI sequences were collected and analyzed: 2 in sinus rhythm, 2

4 in epicardial pacing, and 2 in endocardial pacing. EWI sequences were each made by a 35 recordings of the zone (= 18 loops). In sinus rhythm, visual qualitative displays of the wave

6 front scheme were similar between all recordings: the EWI-induced wave front systematically 7 originated from the endocardium and mid myocardium and moved towards the epicardium 8 with a wide and global displacement (Figure 3). During epicardial and endocardial pacing, 9 visual qualitative displays of the wave front demonstrated a focal positive displacement after 10 rising of the local electrical signal. When visible, the origin of the EWI activation matched the 11 location of the epicardial or endocardial leads (Figures 4 and 5).

13 From the last two hearts, 18 EWI sequences were collected: 6 in sinus rhythm, 6 in epicardial pacing, and 6 in endocardial pacing. EWI sequences were each made by a 3-recordings of the zone (= 54 loops). Every sequence in sinus rhythm, epicardial pacing, and endocardial pacing were all collected 2 times on the anterior, lateral wall, and posterior walls. Two independent readers analyzed all sequences to provide descriptive statistics $(36$ lectures $=108$ loops analyzed).

In spontaneous sinus rhythm, the mean $\pm \mathrm{SD}$ heart rate was $119 \pm 6 \mathrm{bpm}$; the epicardium and endocardium sides were sequentially paced at a mean \pm SD rate of $138 \pm 9 \mathrm{bpm}$.

22 EWI sequences readings matched the electrical activation in $89 \%$ of cases (Table 1). The overall agreement rate between the two readers was $83 \%$. 
1 The readers differentiated in $100 \%$ of cases, paced from sinus rhythm activations, on the

2 anterior, the lateral, and the posterior walls. Mean \pm SD propagation speed of the mechanical

3 wave front in sinus rhythm, into the thickness of the muscle, was $1.7 \pm 0.28 \mathrm{~m} . \mathrm{s}^{-1}$.

4 EWI acquisitions allowed epicardial activation detection in $92 \%$ of cases. When on the

5 posterior or anterior walls, activation patterns from the epicardium were analyzed correctly in

$6100 \%$ of cases. When on the lateral wall, only $75 \%$ of correct analysis was observed (Table

7 1). The mechanical wave front started in a focal zone where the lead was located, and 8 propagated from the epicardium towards the endocardium at a mean $\pm \mathrm{SD}$ speed of $1.3 \pm 0.34$

$9 \mathrm{~m} . \mathrm{s}^{-1}$. EWI acquisitions allowed endocardial activation detection in $75 \%$ of cases. When on 10 the posterior wall, activation patterns detected from the endocardial lead were analyzed 11 correctly in $100 \%$ of cases against $75 \%$ of correct analyses from the lateral wall and $50 \%$ 12 from the anterior wall (Table 1). The mechanical wave front clearly propagated from the endocardium towards the epicardium at a mean \pm SD speed of $1.1 \pm 0.35 \mathrm{~m} \cdot \mathrm{s}^{-1}$. 


\section{Discussion}

2 EWI mapping allows accurate description of a focal ventricular activation

3 Assessment of the activation through electromechanical wave front motion was effective:

4 endocardial versus epicardial stimulation were clearly distinguished using the location of the

5 initial activation and the direction of the wave front propagation. Sinus rhythm was

6 distinguished from paced rhythm using the shape of the wave front and the electromechanical

7 coupling. The present experiment was useful not only to visualize the origin of the

8 mechanical activation within the thickness of a cardiac wall, but also to provide data on the

9 behavior of the wave front into the muscle. Interferences that could jeopardize proper

10 interpretation were distinguished from the electromechanical activation by using the 3-times

11 recorded loops method. Although such a description is still in experimental phase, EWI

12 mapping is promising since the localization of intra myocardial foci may be critical during

13 catheter ablation procedures. It may provide real-time mechanistic insights by linking the

14 wave front speed to histological characteristics of the substrate and be complementary to

15 electro-anatomical mapping systems already available. This technique, more accurate, might

16 help in differentiating the origin of a VT (endocardial versus epicardial) and the underlying 17 mechanism (focal versus reentrance). So far, not a single reliable system enables such 18 observations.

Technical concerns and observations

20 In order to place pacemaker leads easily, at the endocardial and epicardial sides of the three

21 LV portions, experiments were conducted on an isolated working heart instead of a standard

22 in vivo setup. When placing the US probe against the heart, such installation avoids any

23 acoustic fences that can jeopardize the high-resolution activation recordings using an extra 
1 cardiac probe. Since the recordings were performed under a time frame of $200 \mathrm{~ms}$ and the

2 initiation of the EWI was visible during less than 50ms, global heart motion did not

3 jeopardize visualization. Even though the $15 \mathrm{MHz}$ probe used prevented a large visual field

4 (only $14 \mathrm{~mm}$ x $14 \mathrm{~mm}$ square), it offered an accurate view of the muscle. Konofagou's group

5 recently demonstrated a correlation between EWI activation and global electrical activation in

6 a ventricle(Grondin et al. 2016)'(Costet et al. 2016)'(Provost et al. 2011). Their method

7 provides global activation mapping during sinus rhythm(Melki et al. 2017) or stimulated

8 rhythm(Bunting et al. 2017)'(Costet et al. 2016). More recently, epicardial and endocardial

9 pacing were differentiated in $86 \%$ of cases(Costet et al. 2018) but results were based on only

10 eight measurements and did not take into account activation in sinus rhythm. The experiment

11 presented herein confirmed the possibility to differentiate between endocardial and epicardial

12 focal activation with similar rates and adds a complete analysis of the different segments of

13 the LV. Moreover, it allowed measuring of the wave front speed.

14 Finally, the loop-recorded images were confined to a small area but this technique will 15 eventually be able to provide data about a region of interest, once a 3-dimensionnal electro16 anatomical mapping is performed.

Is there a correlation between myocardial structure and EWI?

20 Different activation wave speeds were observed. The large difference between mechanical 21 and electrical waves reported to be around $0.5 \mathrm{~m} \cdot \mathrm{s}^{-1}$ in human hearts(Durrer et al. 1970) points 22 to the strong possibility that a mechanical wave - once initiated by the "kick" of an electrical 23 stimulus - mainly depends on the mechanical properties of the tissue. Wave front speed 24 changes could be related to the orientation of the myocardial fibers that follow different 
1 directions before reaching the Purkinje system and may help to determine new properties of

2 the cardiac muscle.

3 Even though spatial resolution of the sequence presented herein prevents high-resolution

4 analysis of the activated wave front shape and speed, this technique might support the in vivo 5 correlation of anisotropic properties with the orientations of epicardial and endocardial 6 fibers(Deng et al. 2012), the anatomy of the Purkinje that usually contains more denser fibers 7 close to the endocardium(Garcia-Bustos et al. 2017), and the dispersion of the gap junction 8 density from the endocardium towards the epicardium(Yamada et al. 2004). Further 9 evaluations will be needed, combining information from a 3-dimensional histological analysis 10 of the interlaced fibers and a 3-dimensional ultrasound analysis of the area.

\section{Limitations}

The present study has some limitations. All images were obtained in swine hearts, in an isolated bi-ventricular working mode with a 2-D probe. Results were therefore based on mechanical recordings from a 2 dimensional space of an artificial endocardial and epicardial paced activation. Moreover, since the hearts were healthy, no pathological substrate was present inside the muscle. Even though 2-D spatial analyses sometimes prevented the wave front to be clearly visible, the propagation of the electromechanical waves was looking homogenous making the identification of the paced site easier and the calculation of the mechanical wave front speed possible. Interestingly, global EWI mapping has already been performed in sinus rhythm of myocardial infarction models(Costet et al. 2017), and on ablated

23 myocardium(Papadacci et al. 2017a). Since substrate analysis was successfully achieved in 24 those models, clinical application should be attainable. However, VT would induce more 25 complex schemes of activation in the three dimensions. Future experiments are needed using 
1 a three-dimensional ultrasound and pathological models to determine the local EWI into a

2 scar and the ability of the system to determine a reentrant circuit.

3

$4 \quad$ Ultrasound based mapping and its part in the future of electrophysiology

6 Since the understanding of underlying mechanisms during VT ablation is one of the 7 cornerstones of a successful procedure, the type of acquisition presented here could be 8 integrated with electro-anatomical data, during catheter-based procedure, in order to improve 9 ablation success. This sequence could be embedded into an intra-cardiac echography (ICE) 10 system, avoiding any acoustic fences that could compromise quality of the images. Since ICE 11 already offers 2-D images and should be providing 3-D images in the next decade, the 12 addition of such sequences should allow a real-time "single beat" EWI activation map of 13 interlaced myocardial fibers inside an arrhythmic ventricle wall(Papadacci et al. 2017b). 14 Another option to limit acoustic fences would consist in providing such sequences under 15 transesophageal echocardiography (TEE) analysis, which already offers 2-D and 3-D 16 acquisitions. The combination of EWI mapping with elastography (available with ICE and 17 TEE) could provide both the location of the arrhythmia and the characteristics (shape, surface, volume) of the thermal lesion after the ablation(Kwiecinski et al. 2015)'(Kwiecinski et al. 2014)'(Sandoval et al. 2018). Since high-intensity focused ultrasounds can be delivered from a TEE(Bessiere et al. 2016)'(Constanciel et al. 2013) into the ventricles, ultrasound energy 21 might be able, in the future, to localize, treat, and monitor cardiac arrhythmias. 
3 EWI mapping allows assessment of mechanical activation of a LV wall in healthy hearts of an

4 isolated working mode system. Endocardial and epicardial pacing can be easily differentiated 5 from sinus rhythm activation and EWI-induced wave front can be accurately analyzed. This 6 technique is promising to provide help in differentiating between endocardial and epicardial 7 arrhythmias, during complex ablation procedures, and to characterize the mechanism of 8 ventricular activation. Future experiments will need to be conducted in diseased hearts, and 9 clinical data will be needed to correlate electrophysiological data to EWI activation patterns.

\section{Acknowledgements}

19 This work was supported by the French National Research Agency (ANR) under the

20 CHORUS Grant project ANR17-CE19-0017 and France Life Imaging - WP3 2019. Dr

21 Verena Landel is warmly thanked for her contribution and proofreading of the manuscript. 
3 Bessiere F, N'djin WA, Colas EC, Chavrier F, Greillier P, Chapelon JY, Chevalier P, Lafon

4 C. Ultrasound-Guided Transesophageal High-Intensity Focused Ultrasound Cardiac Ablation 5 in a Beating Heart: A Pilot Feasibility Study in Pigs. Ultrasound Med Biol 2016;42:184861861.

7 Bunting E, Lambrakos L, Kemper P, Whang W, Garan H, Konofagou E. Imaging the 8 Propagation of the Electromechanical Wave in Heart Failure Patients with Cardiac 9 Resynchronization Therapy. Pacing Clin Electrophysiol PACE 2017;40:35-45.

Constanciel E, N'Djin WA, Bessière F, Chavrier F, Grinberg D, Vignot A, Chevalier P,

11 Chapelon JY, Lafon C. Design and evaluation of a transesophageal HIFU probe for 12 ultrasound-guided cardiac ablation: simulation of a HIFU mini-maze procedure and preliminary ex vivo trials. IEEE Trans Ultrason Ferroelectr Freq Control 2013;60:1868-1883.

Konofagou E. Electromechanical wave imaging and electromechanical wave velocity estimation in a large animal model of myocardial infarction. Phys Med Biol 2017;62:93419356.

Costet A, Wan E, Bunting E, Grondin J, Garan H, Konofagou E. Electromechanical wave imaging (EWI) validation in all four cardiac chambers with 3D electroanatomic mapping in canines in vivo. Phys Med Biol 2016;61:8105-8119.

Costet A, Wan E, Melki L, Bunting E, Grondin J, Garan H, Konofagou E. Non-invasive Characterization of Focal Arrhythmia with Electromechanical Wave Imaging in Vivo.

23 Ultrasound Med Biol 2018;

24 Deng D, Jiao P, Ye X, Xia L. An image-based model of the whole human heart with detailed 25 anatomical structure and fiber orientation. Comput Math Methods Med 2012;2012:891070. 
1 Durrer D, van Dam RT, Freud GE, Janse MJ, Meijler FL, Arzbaecher RC. Total excitation of

2 the isolated human heart. Circulation 1970;41:899-912.

3 Enriquez A, Malavassi F, Saenz LC, Supple G, Santangeli P, Marchlinski FE, Garcia FC.

4 How to map and ablate left ventricular summit arrhythmias. Heart Rhythm 2017;14:141-148.

5 Erkapic D, Greiss H, Pajitnev D, Zaltsberg S, Deubner N, Berkowitsch A, Möllman S,

6 Sperzel J, Rolf A, Schmitt J, Hamm CW, Kuniss M, Neumann T. Clinical impact of a novel

7 three-dimensional electrocardiographic imaging for non-invasive mapping of ventricular

8 arrhythmias-a prospective randomized trial. Eur Eur Pacing Arrhythm Card Electrophysiol J

9 Work Groups Card Pacing Arrhythm Card Cell Electrophysiol Eur Soc Cardiol 2015;17:59110597.

11 Fernandez-Armenta J, Berruezo A. How to recognize epicardial origin of ventricular 12 tachycardias? Curr Cardiol Rev 2014;10:246-256.

13 Garcia-Bustos V, Sebastian R, Izquierdo M, Molina P, Chorro FJ, Ruiz-Sauri A. A

14 quantitative structural and morphometric analysis of the Purkinje network and the Purkinje15 myocardial junctions in pig hearts. J Anat 2017;230:664-678.

16 Grondin J, Costet A, Bunting E, Gambhir A, Garan H, Wan E, Konofagou EE. Validation of 17 Electromechanical Wave Imaging in a canine model during pacing and sinus rhythm. Heart 18 Rhythm Off J Heart Rhythm Soc 2016;13:2221-2227.

19 J Shah A, Hocini M, Pascale P, Roten L, Komatsu Y, Daly M, Ramoul K, Denis A, Derval N,

20 Sacher F, Dubois R, Bokan R, Eliatou S, Strom M, Ramanathan C, Jais P, Ritter P,

21 Haissaguerre M. Body Surface Electrocardiographic Mapping for Non-invasive Identification

22 of Arrhythmic Sources. Arrhythmia Electrophysiol Rev 2013;2:16-22.

23 Kwiecinski W, Bessière F, Colas EC, N'Djin WA, Tanter M, Lafon C, Pernot M. Cardiac 24 shear-wave elastography using a transesophageal transducer: application to the mapping of 25 thermal lesions in ultrasound transesophageal cardiac ablation. Phys Med Biol 2015;60:7829_ 
2 Kwiecinski W, Provost J, Dubois R, Sacher F, Haïssaguerre M, Legros M, Nguyen-Dinh A,

3 Dufait R, Tanter M, Pernot M. Quantitative evaluation of atrial radio frequency ablation using

$4 \quad$ intracardiac shear-wave elastography. Med Phys 2014;41:112901.

5 Lerman BB. Mechanism, diagnosis, and treatment of outflow tract tachycardia. Nat Rev

6 Cardiol 2015;12:597-608.

7 Melki L, Costet A, Konofagou EE. Reproducibility and Angle Independence of

8 Electromechanical Wave Imaging for the Measurement of Electromechanical Activation

9 during Sinus Rhythm in Healthy Humans. Ultrasound Med Biol 2017;43:2256-2268.

10 Nguyen T-M, Song S, Arnal B, Huang Z, O’Donnell M, Wang RK. Visualizing ultrasonically

11 induced shear wave propagation using phase-sensitive optical coherence tomography for

12 dynamic elastography. Opt Lett 2014;39:838-841.

13 Papadacci C, Bunting EA, Wan EY, Nauleau P, Konofagou EE. 3D myocardial elastography

14 and electromechanical wave imaging in vivo. IEEE Trans Med Imaging 2017a;36:618-627.

15 Papadacci C, Finel V, Provost J, Villemain O, Bruneval P, Gennisson J-L, Tanter M, Fink M,

16 Pernot M. Imaging the dynamics of cardiac fiber orientation in vivo using 3D Ultrasound

17 Backscatter Tensor Imaging. Sci Rep 2017b;7:830.

18 Pinton GF, Dahl JJ, Trahey GE. Rapid tracking of small displacements with ultrasound. IEEE

19 Trans Ultrason Ferroelectr Freq Control 2006;53:1103-1117.

20 Provost J, Lee W-N, Fujikura K, Konofagou EE. Imaging the electromechanical activity of

21 the heart in vivo. Proc Natl Acad Sci U S A 2011;108:8565-8570.

22 Revishvili AS, Wissner E, Lebedev DS, Lemes C, Deiss S, Metzner A, Kalinin VV, Sopov

23 OV, Labartkava EZ, Kalinin AV, Chmelevsky M, Zubarev SV, Chaykovskaya MK, Tsiklauri

24 MG, Kuck K-H. Validation of the mapping accuracy of a novel non-invasive epicardial and

25 endocardial electrophysiology system. Eur Eur Pacing Arrhythm Card Electrophysiol J Work 
1 Groups Card Pacing Arrhythm Card Cell Electrophysiol Eur Soc Cardiol 2015;17:1282-1288.

2 Sandoval Z, Castro M, Alirezaie J, Bessière F, Lafon C, Dillenseger J-L. Transesophageal 2D

3 ultrasound to 3D computed tomography registration for the guidance of a cardiac arrhythmia

$4 \quad$ therapy. Phys Med Biol 2018;63:155007.

5 Shivkumar K. Catheter Ablation of Ventricular Arrhythmias. N Engl J Med 2019;380:1555-

61564.

7 Vaillant F, Magat J, Bour P, Naulin J, Benoist D, Loyer V, Vieillot D, Labrousse L, Ritter P,

8 Bernus O, Dos Santos P, Quesson B. Magnetic resonance-compatible model of isolated

9 working heart from large animal for multimodal assessment of cardiac function,

10 electrophysiology, and metabolism. Am J Physiol Heart Circ Physiol 2016;310:H1371-1380.

11 Yamada KA, Kanter EM, Green KG, Saffitz JE. Transmural distribution of connexins in

12 rodent hearts. J Cardiovasc Electrophysiol 2004;15:710-715.

13

14

15

16

17

18

19

20

21

22

23

24 
1 Table 1 Summary of the correct Electromechanical Wave Imaging (EWI) sequences

2 interpretations from two independent readers

\begin{tabular}{|c|c|c|c|c|c|c|}
\hline \multicolumn{3}{|c|}{ Activation origin } & \multirow{2}{*}{$\begin{array}{c}\text { EPICARDIUM } \\
\text { 3/4 }\end{array}$} & \multirow{2}{*}{$\begin{array}{l}\text { ENDOCARDIUM } \\
\qquad 3 / 4\end{array}$} & \multirow{2}{*}{$\begin{array}{c}\text { SINUS } \\
\text { RHYTHM } \\
4 / 4\end{array}$} & \multirow{2}{*}{$\begin{array}{l}\text { TOTAL } \\
10 / / 12\end{array}$} \\
\hline \multirow{8}{*}{ SEGMENT } & \multirow{2}{*}{ LATERAL } & $\mathrm{n}$ & & & & \\
\hline & & $\%$ & $75 \%$ & $75 \%$ & $100 \%$ & $83 \%$ \\
\hline & \multirow{2}{*}{ POSTERIOR } & $\mathrm{n}$ & $4 / 4$ & $4 / 4$ & $4 / 4$ & $12 / 12$ \\
\hline & & $\%$ & $100 \%$ & $100 \%$ & $100 \%$ & $100 \%$ \\
\hline & \multirow{2}{*}{ ANTERIOR } & $\mathrm{n}$ & $4 / 4$ & $2 / 4$ & $4 / 4$ & $10 / 12$ \\
\hline & & $\%$ & $100 \%$ & $50 \%$ & $100 \%$ & $83 \%$ \\
\hline & \multirow{2}{*}{ TOTAL } & $\mathrm{n}$ & $11 / 12$ & $9 / 12$ & $12 / 12$ & $32 / 36$ \\
\hline & & $\%$ & $92 \%$ & $75 \%$ & $100 \%$ & $89 \%$ \\
\hline
\end{tabular}

3

4

5

6

7

8

9

10

11

12

13

14

15

16

17 


\section{Figure Captions}

2

3 Figure 1: Isolated working mode heart. The $15 \mathrm{MHz}$ ultrasound (US) probe is facing the left

4 ventricle (LV). Two leads are screwed into the epicardial and endocardial sides of the LV.

5 The one on the endocardial side is placed through the left atrium, from a pulmonary vein.

6

7 Figure 2: The cross-cut view of the left ventricle depicts the relation between the epicardial 8 and endocardial leads and the ultrasound probe (short-axis).

9 the epicardial lead.

Figure 3: Electromechanical wave imaging (EWI) in sinus rhythm. The first column shows the electrocardiogram: from the first to the last line, a red dot depicts when the images were recorded at four different moments of a cardiac cycle (ms). The second column shows the Bmode corresponding images. The third column shows the corresponding EWI pictures (deformation depicted in $\mu \mathrm{m}$ ). The beginning of the induced electromechanical wave front is wide, originating from the endocardium towards the epicardium.

Figure 4: EWI during epicardial pacing. The first column shows the electrocardiogram: from the first to the last line, a red dot depicts when the images were recorded at four different moments of a cardiac cycle (ms). The second column shows the B-mode corresponding images. The third column shows the corresponding EWI pictures (deformation depicted in $\mu \mathrm{m})$. The beginning of the induced electromechanical wave front is focal and clearly distinguished on the upper right corner of the image (in red). It corresponds to the location of 
1 Figure 5: EWI during endocardial pacing. The first column shows the electrocardiogram:

2 from the first to the last line, a red dot depicts when the images were recorded at four different 3 moments of a cardiac cycle (ms). The second column shows the B-mode corresponding 4 images. The third column shows the corresponding EWI pictures (deformation depicted in $5 \mu \mathrm{m})$. The beginning of the induced electromechanical wave front is focal and clearly 6 distinguished on the lower left corner of the image (in red). It corresponds to the location of 7 the endocardial lead.

8 


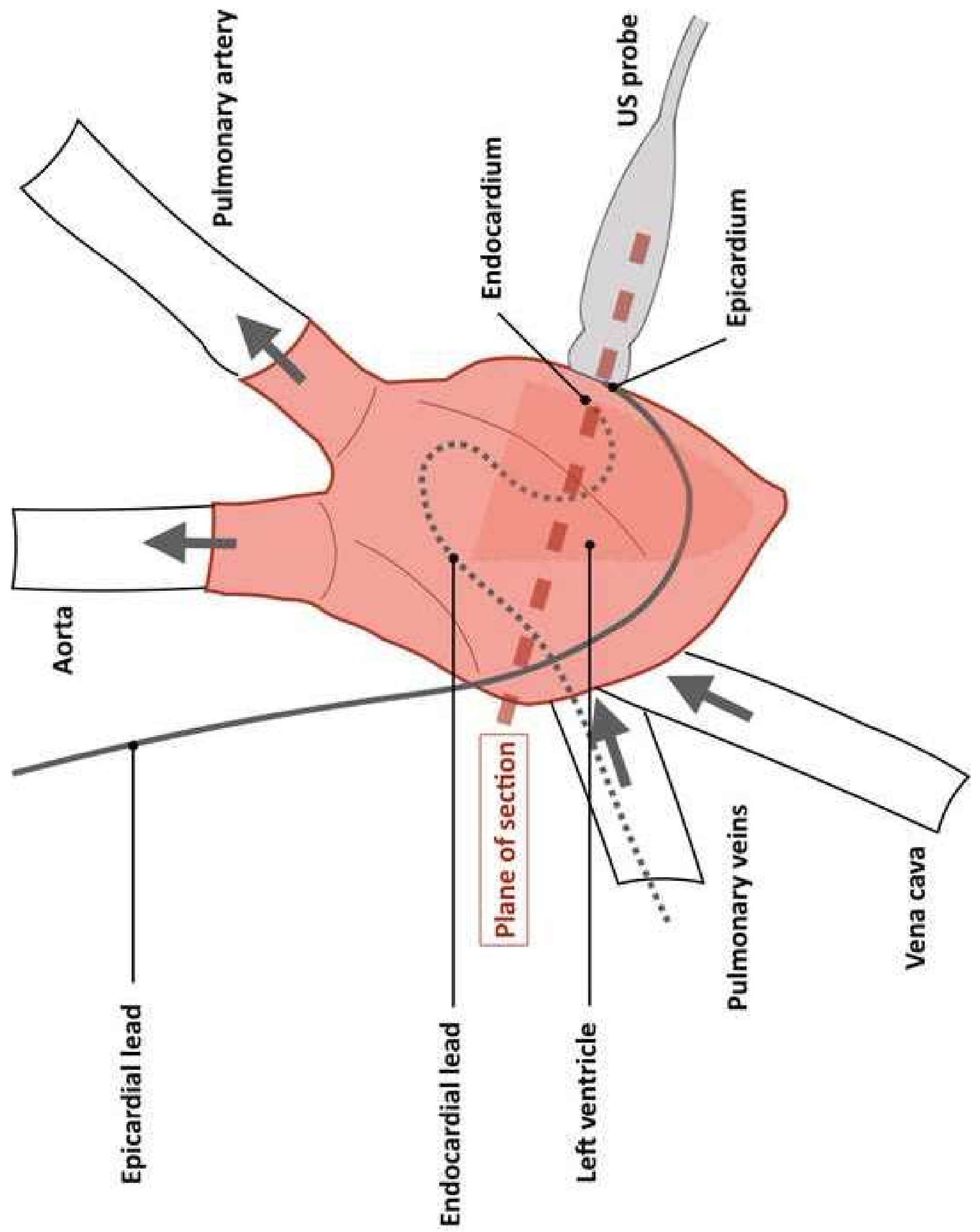

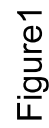




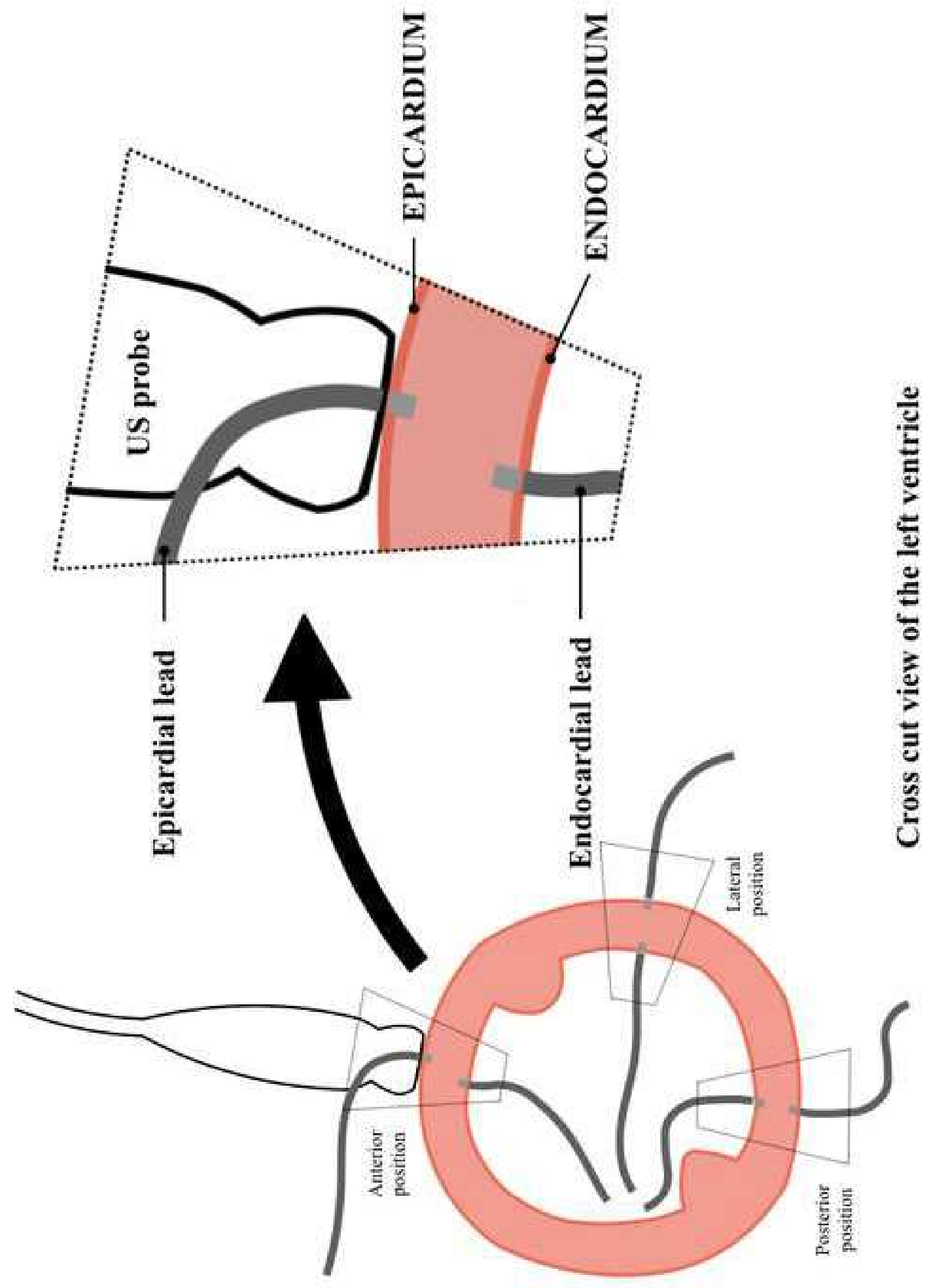

$\frac{\text { }}{\frac{d}{3}}$ 

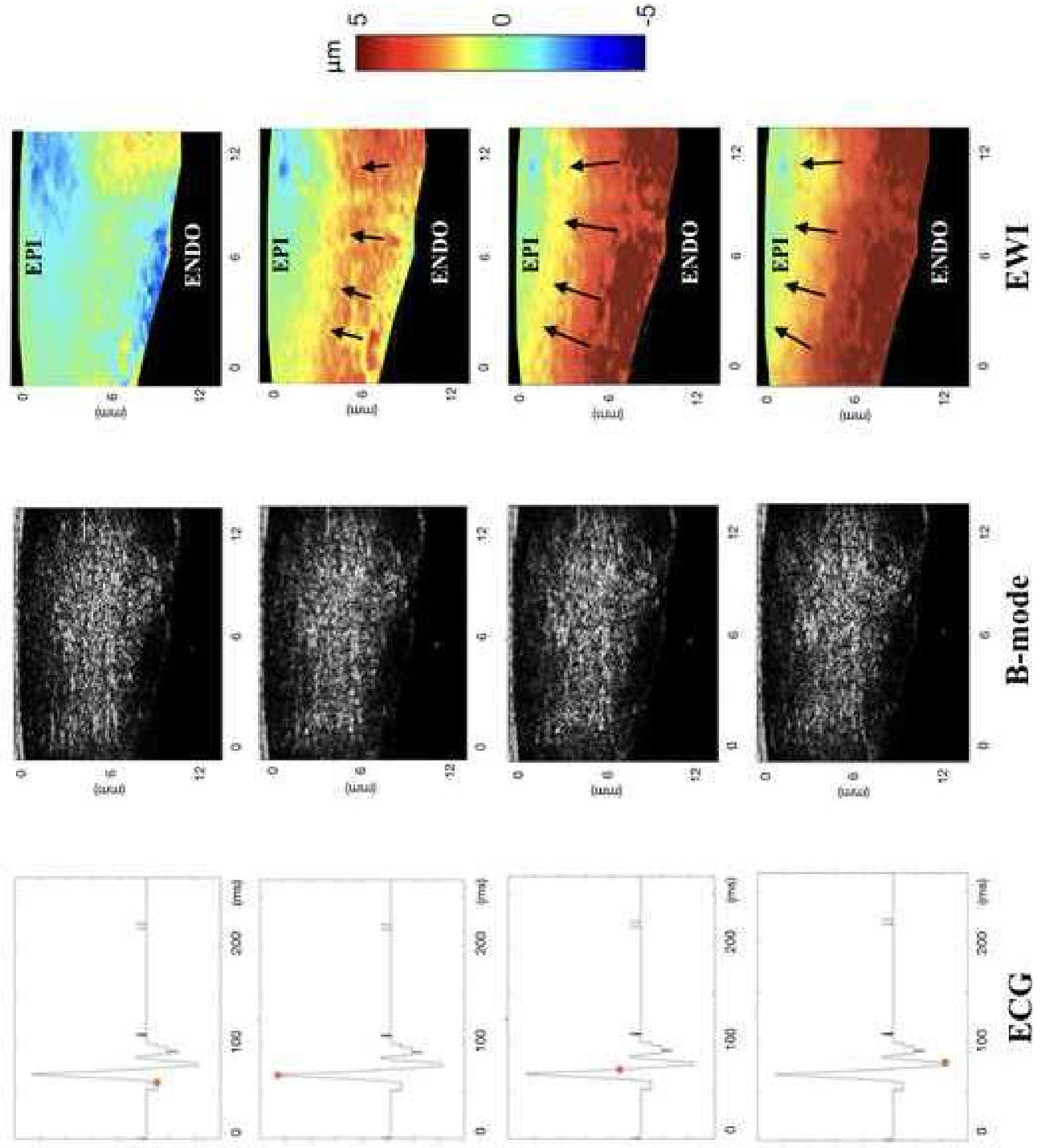

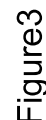

N

$\infty$

$\forall$ 
1

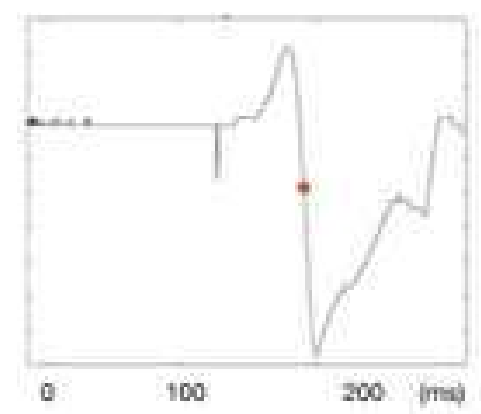

2

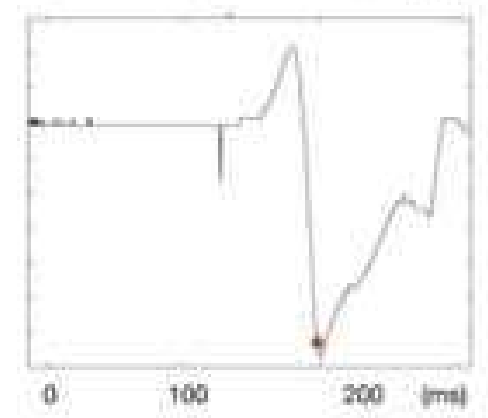

3

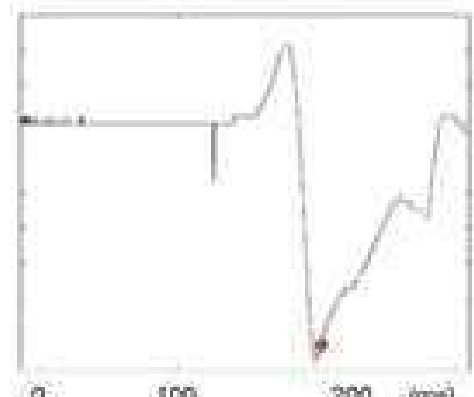

4

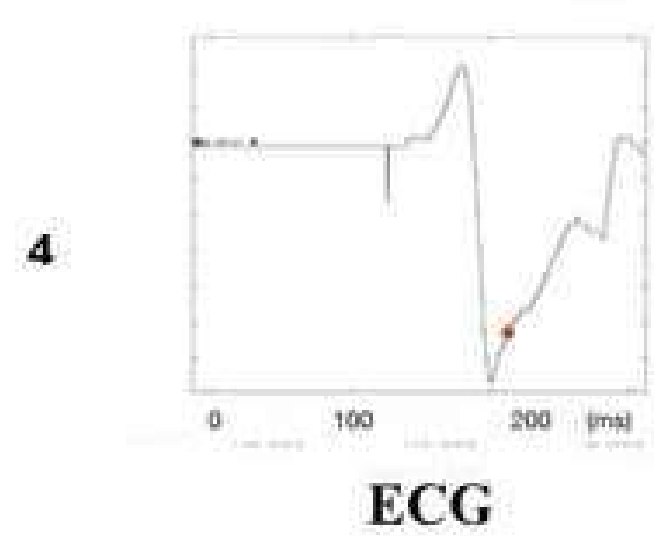

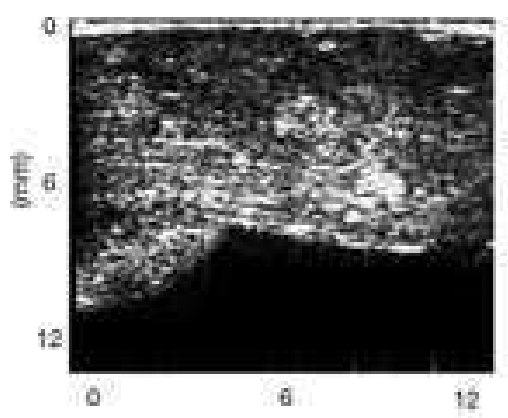
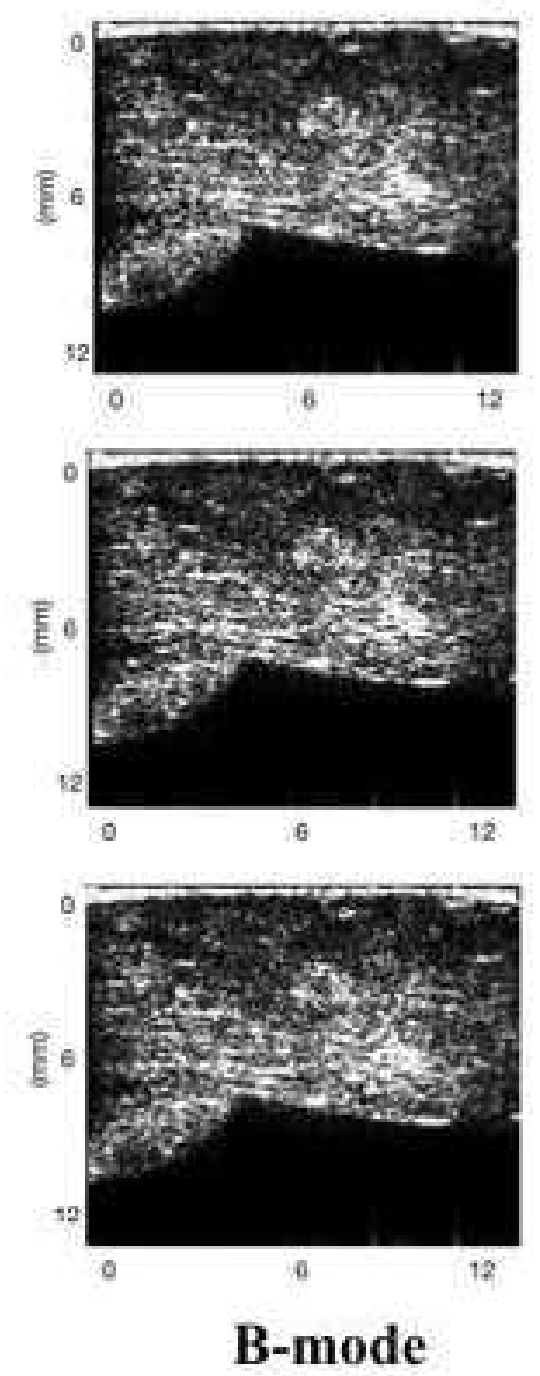

B-mode
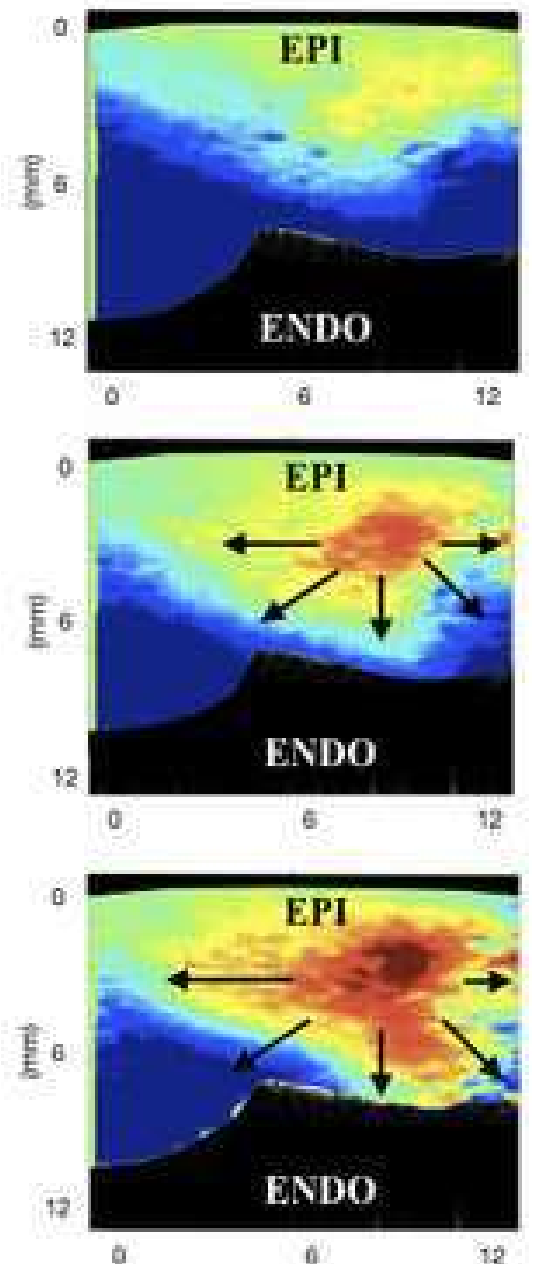

EWI
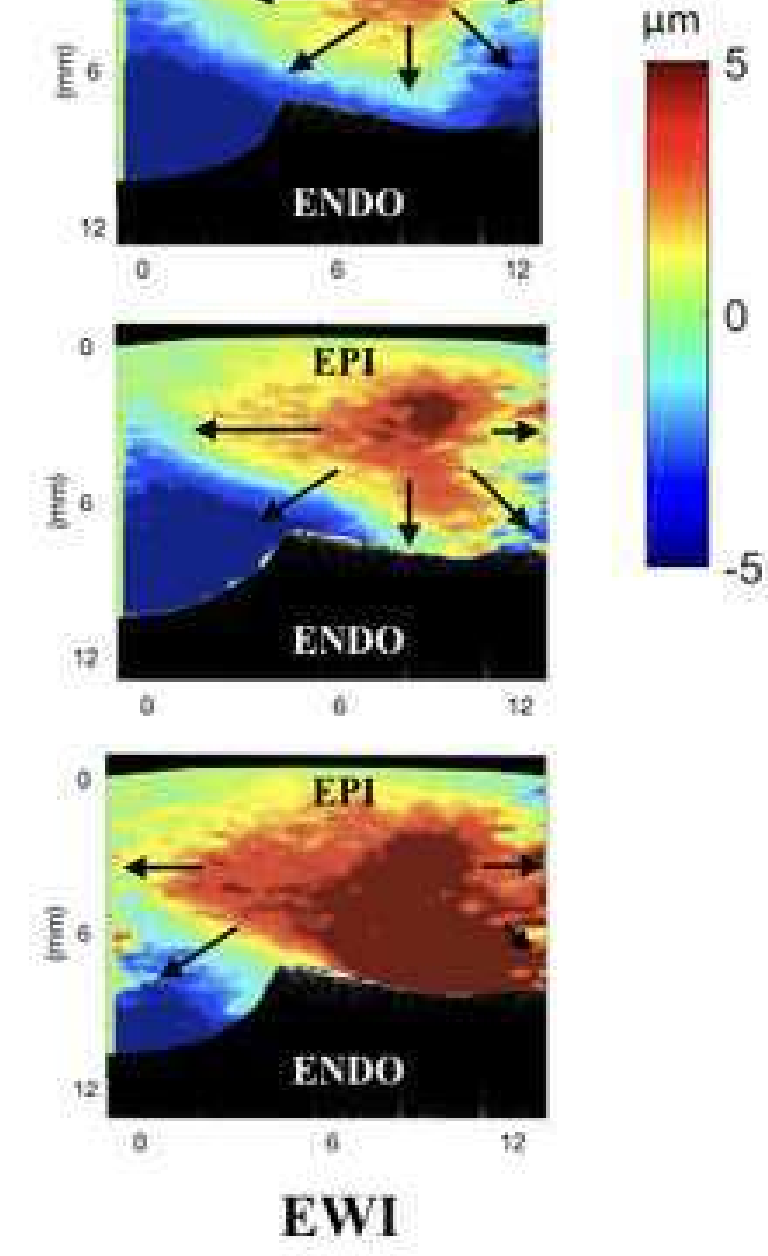
1

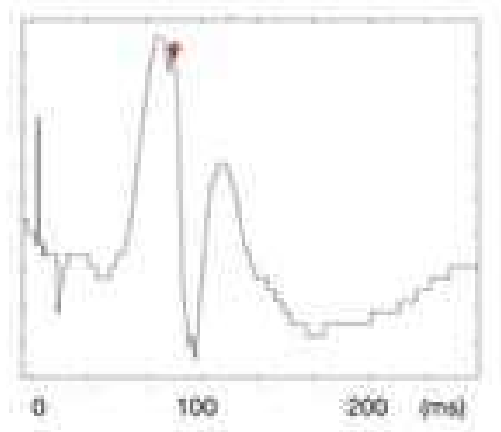

2

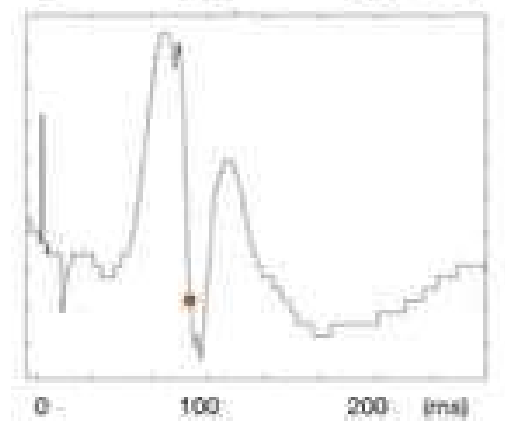

3

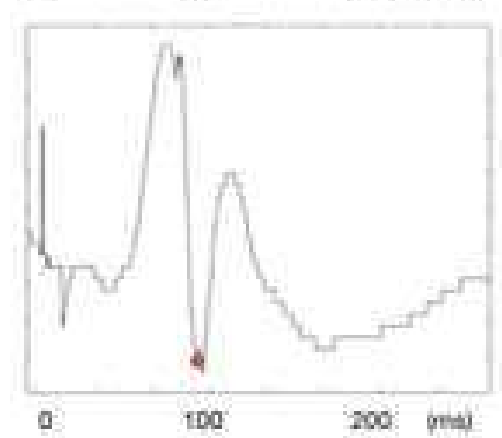

4

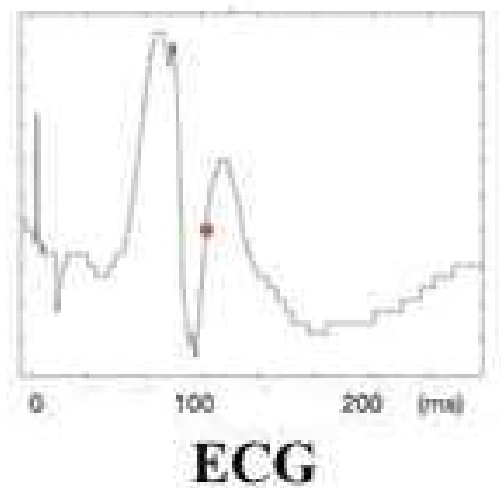

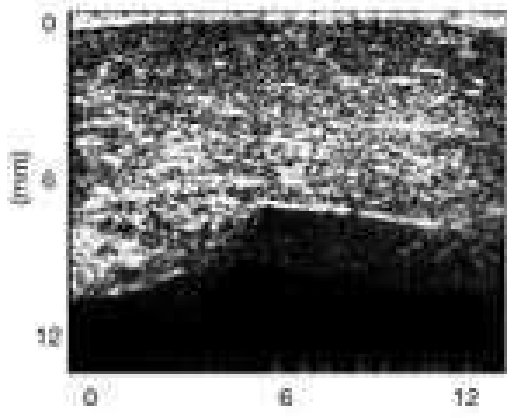
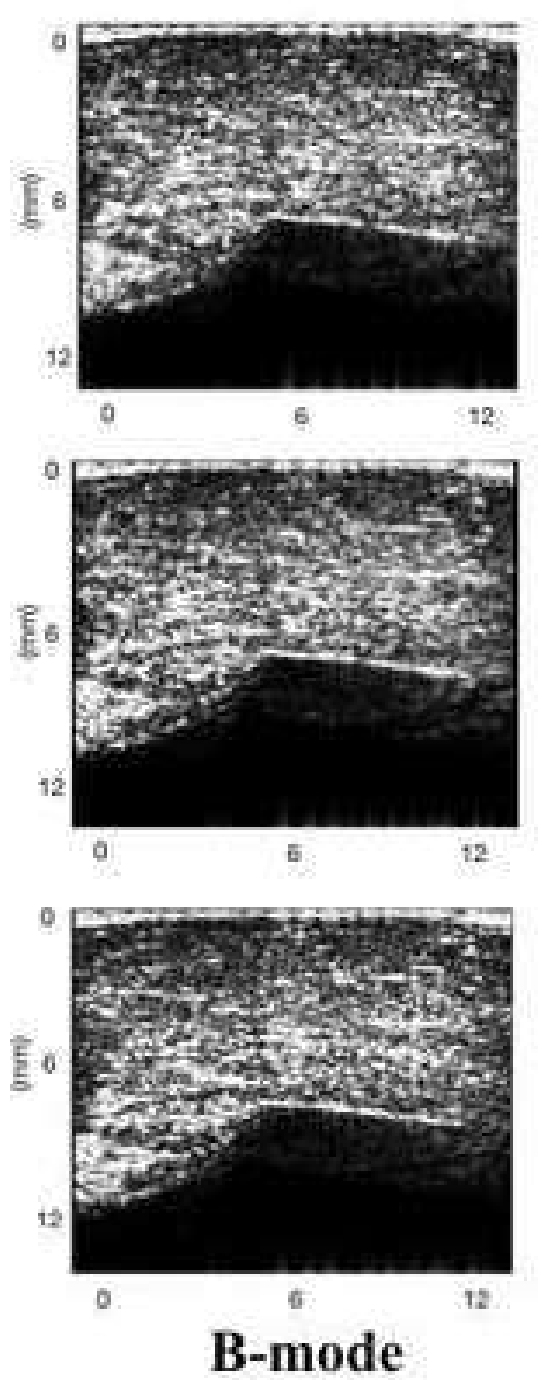
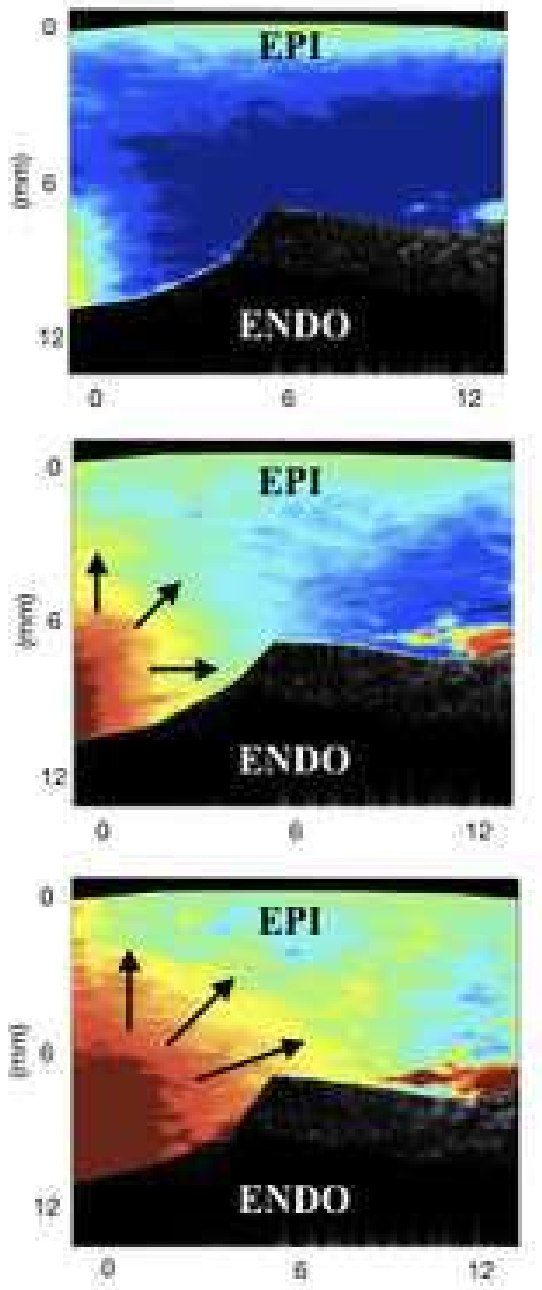

$\mu \mathrm{m}$

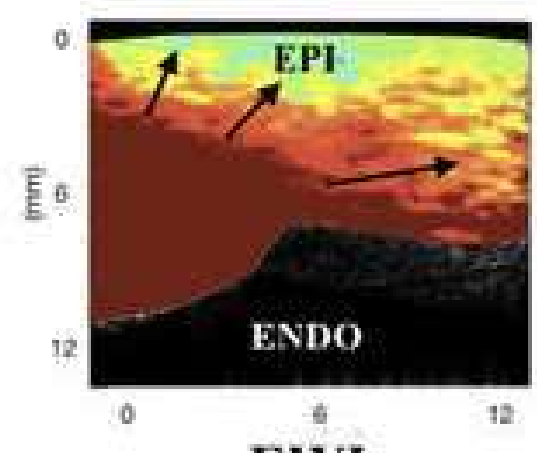

EWI

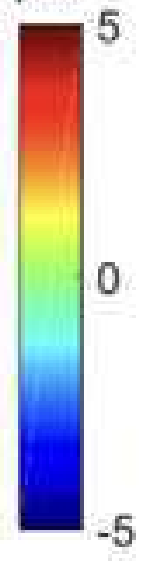

